\title{
13. NEOGENE DIATOMS FROM THE EMPEROR SEAMOUNT CHAIN, LEG 55, DEEP SEA DRILLING PROJECT
}

\author{
Itaru Koizumi, Institute of Geological Sciences, College of General Education, \\ Osaka University, Toyonaka, Osaka, Japan
}

\section{INTRODUCTION}

Leg 55 of the Deep Sea Drilling Project started from Honolulu, Hawaii, on 23 July 1977 and terminated at Yokohama, Japan on 6 September, 1977. Eleven holes were drilled at 4 sites; a multiple re-entry hole was drilled on Suiko Seamount (Figure 1). As originally planned, Leg 55 had two objectives: (1) drilling at least two sites on the Emperor Seamount chain, and (2) drilling a third site in Mesozoic Pacific crust in anomaly M-1 on the oceanic side of the Kuril trench. The proposed site in Mesozoic crust, however, was abandoned for logistical reasons. Because the principal objective of Leg 55 was to test the "hot-spot" hypothesis of the origin of the Hawaiian and Emperor chains, the recovery of sedimentary sequences for diatoms was not of primary importance. Drilling on Suiko Seamount (Site 433), however, yielded pelagic sediments which consist mostly of skeletons of diatoms, radiolarians, and foraminifers, without the terrigenous material such as sand, silt, and volcanic ash.

Eighty-two samples from the 11 holes drilled on Leg 55 were examined for diatoms. The entire upper Neogene section of the northernmost site (433) contains abundant and moderately well preserved diatoms. Regrettably, diatoms are very rare throughout the samples from Quaternary sediments of the southern sites (Sites 430 to 432 ), owing to the lower primary production in the warm-water area; no diatoms were recovered in any samples from sediments older than lower Neogene from the northernmost site.

\section{PREPARATION OF SAMPLES}

All samples examined were taken on board D/V Glomar Challenger from suitable levels in the core sections. The sample spacing in critical intervals was sufficiently close that stratigraphic first and last occurrences of selected species were determined with reasonable accuracy.

Original wet material of about 1 gram weight was dropped into a $200-\mathrm{ml}$ beaker of boiling hydrogen peroxide solution $\left(\mathrm{H}_{2} \mathrm{O}_{2}, 15 \%\right)$. Upon cooling, hydrochloric acid was added to make an acid solution (approximately $25 \%$ in $\mathrm{HCl}$ by volume) that was then boiled. After boiling for about 5 minutes, the beaker was filled with distilled water and allowed to stand for 4 hours. Then the residue was separated by decanting the acid water in the beaker. The beaker was filled again with distilled water, and this decanting process was repeated four times.

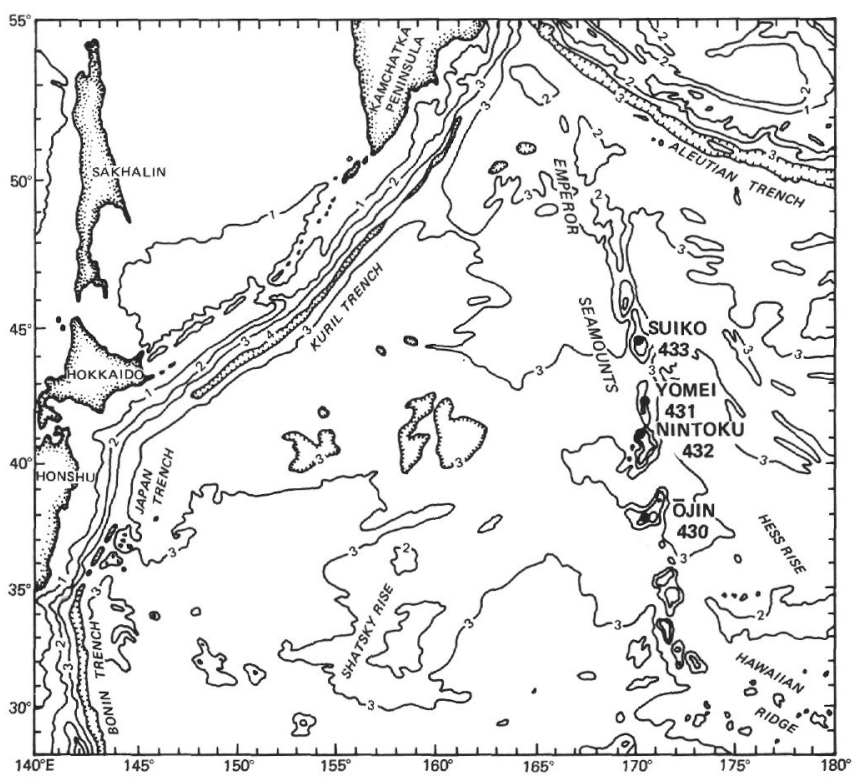

Figure 1. Location of drilling sites occupied on DSDP Leg 55, the northwestern Pacific Ocean. Contour depths are in kilometers (from map "Topography of North Pacific" by T. E. Chase, H. W. Menard, and J. Mammerickx, Institute of Marine Resources, Geologic Data Center, Scripps Institution of Oceanography, 1971).

The residue was stirred while adding distilled water. After about 10 seconds, when clastic grains and volcanic glass sank to the lower part or the bottom of the beaker, approximate quantities of the suspension containing diatom valves were taken with a $1-\mathrm{ml}$ pipette from the middle part of the water column in the beaker, and placed on a square cover glass $(18 \times 18 \mathrm{~mm}$ in size). The cover glass was dried on a hot plate at moderate temperature (about $60^{\circ}$ to $80^{\circ} \mathrm{C}$ ) and then mounted on a slide glass using Aroclor No. 4465 (refractive index 1.66).

\section{METHOD OF STUDY}

Investigations were carried out using a Carl Zeiss Standard RA microscope with a lens combination of $\mathrm{kpl}$ W12.5 $\times$ and Plan Oil $100 \times / 1.25$. All diatom species were identified and counted throughout the samples from Sites 430, 431, and 432 (Tables 1 to 3). The single count of 200 specimens was done for the samples from Site 433 (Table 4). The microscope work was completed by adding 200 more specimens in order to know the oc- 
TABLE 1

Neogene Diatoms

\begin{tabular}{|c|c|c|c|c|c|c|c|c|c|c|c|c|c|c|c|c|c|c|}
\hline & \multicolumn{7}{|c|}{ DSDP, Leg 55, Site 430} & \multirow[b]{2}{*}{ 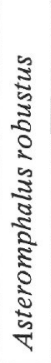 } & \multirow[b]{2}{*}{ 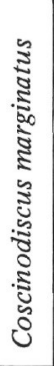 } & \multirow[b]{2}{*}{ 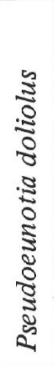 } & \multirow[b]{2}{*}{ 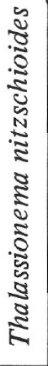 } & \multirow[b]{2}{*}{ 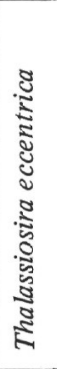 } & \multirow[b]{2}{*}{ 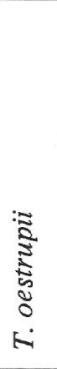 } & \multirow[b]{2}{*}{ 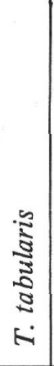 } & \multirow[b]{2}{*}{ 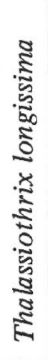 } & \multirow[b]{2}{*}{ 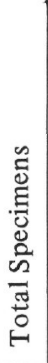 } & \multirow[b]{2}{*}{ 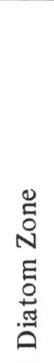 } & \multirow[b]{2}{*}{ 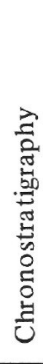 } \\
\hline$\frac{0}{\frac{0}{1}}$ & 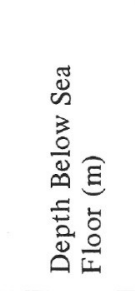 & 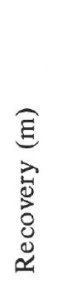 & نั & 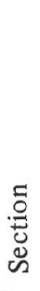 & 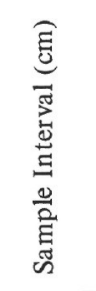 & 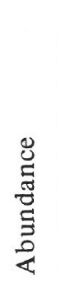 & 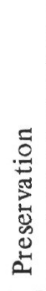 & & & & & & & & & & & \\
\hline & $2.5-4.5$ & 7.7 & 2 & $\begin{array}{l}3 \\
5\end{array}$ & $\begin{array}{c}10-20 \\
30-35 \\
\text { CC } \\
\end{array}$ & $\begin{array}{l}\text { VR } \\
\text { VR } \\
\text { B }\end{array}$ & $\begin{array}{l}\mathrm{p} \\
\mathrm{p}\end{array}$ & 1 & 1 & 1 & 1 & 1 & 1 & & & $\begin{array}{l}3 \\
3\end{array}$ & ن. & $\frac{\dot{\omega}}{a}$ \\
\hline A & $\begin{array}{c}0-5.5 \\
19.0-28.5\end{array}$ & 1.0 & 1 & 1 & $\begin{array}{c}25-30 \\
\text { CC } \\
\text { CC }\end{array}$ & $\begin{array}{c}\text { VR } \\
\text { B } \\
\text { B }\end{array}$ & $\mathrm{p}$ & & & 2 & & & 2 & 1 & 2 & 7 & ¿ & i \\
\hline
\end{tabular}

TABLE 2

Neogene Diatoms

\begin{tabular}{|c|c|c|c|c|c|c|c|c|c|c|c|c|c|c|c|c|c|c|c|c|c|}
\hline \multicolumn{8}{|c|}{ DSDP, Leg 55 , Site 431} & \multirow[b]{2}{*}{ 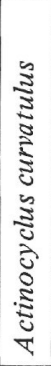 } & \multirow[b]{2}{*}{ 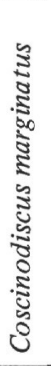 } & \multirow[b]{2}{*}{ 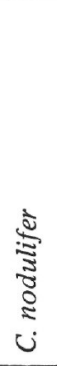 } & \multirow[b]{2}{*}{ 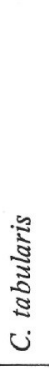 } & \multirow[b]{2}{*}{ 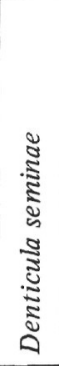 } & \multirow[b]{2}{*}{ 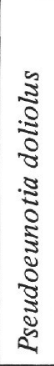 } & \multirow[b]{2}{*}{ 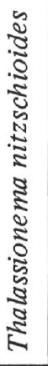 } & \multirow[b]{2}{*}{ 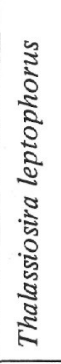 } & \multirow[b]{2}{*}{ 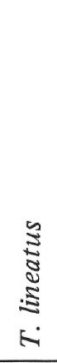 } & \multirow[b]{2}{*}{ 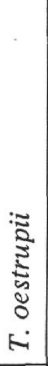 } & \multirow[b]{2}{*}{ 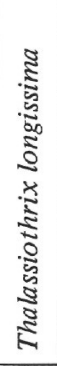 } & \multirow[b]{2}{*}{ 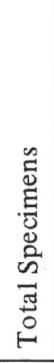 } & \multirow[b]{2}{*}{ 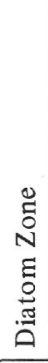 } & \multirow[b]{2}{*}{ 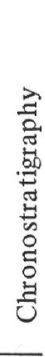 } \\
\hline $\begin{array}{l}\stackrel{0}{0} \\
\text { I }\end{array}$ & 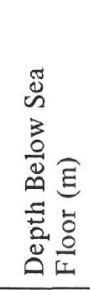 & 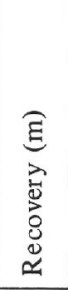 & ن & 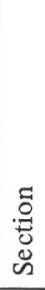 & 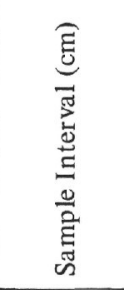 & 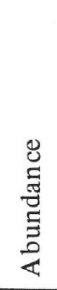 & 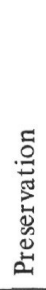 & & & & & & & & & & & & & & \\
\hline & $0-9.5$ & 1.9 & 1 & 1 & $\begin{array}{c}64-66 \\
\text { CC }\end{array}$ & $\begin{array}{l}\text { VR } \\
\text { VR }\end{array}$ & $\begin{array}{l}\mathrm{p} \\
\mathrm{p}\end{array}$ & 1 & 2 & 1 & 1 & $\begin{array}{l}2 \\
7\end{array}$ & 1 & $\begin{array}{l}4 \\
3 \\
\end{array}$ & & 1 & 1 & $\begin{array}{l}1 \\
2 \\
\end{array}$ & $\begin{array}{r}7 \\
20 \\
\end{array}$ & $\dot{a}$ & $\overrightarrow{\vec{a}}$ \\
\hline A & $0-7.5$ & 1.9 & 1 & 1 & $\begin{array}{c}5-8 \\
145-148 \\
\text { CC }\end{array}$ & $\begin{array}{l}\text { VR } \\
\text { VR } \\
\text { VR }\end{array}$ & $\begin{array}{l}\mathrm{p} \\
\mathrm{p} \\
\mathrm{p}\end{array}$ & & 1 & & 1 & $\begin{array}{r}17 \\
1\end{array}$ & 1 & $\begin{array}{l}3 \\
1 \\
1\end{array}$ & 1 & 1 & 2 & 4 & $\begin{array}{r}31 \\
2 \\
1\end{array}$ & $\dot{a}$ & $\frac{\bar{\omega}}{\alpha}$ \\
\hline
\end{tabular}

TABLE 3

Neogene Diatoms

\begin{tabular}{|c|c|c|c|c|c|c|c|c|c|c|c|c|c|c|c|c|c|c|c|c|c|c|c|c|c|c|c|c|c|c|c|c|c|c|}
\hline \multicolumn{7}{|c|}{ DSDP, Leg 55 , Site 432} & \multirow[b]{2}{*}{ 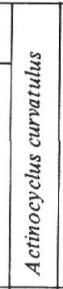 } & & \multirow[b]{2}{*}{ 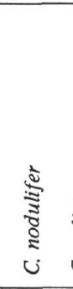 } & \multirow{2}{*}{\multicolumn{2}{|c|}{ 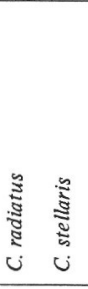 }} & \multirow[b]{2}{*}{ 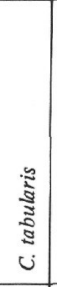 } & \multirow[b]{2}{*}{ 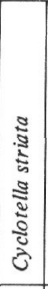 } & \multirow[b]{2}{*}{ 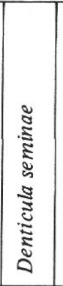 } & \multirow[b]{2}{*}{ 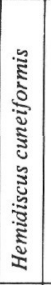 } & \multirow[b]{2}{*}{ 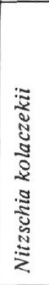 } & \multirow[b]{2}{*}{ 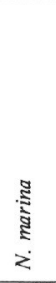 } & \multirow[b]{2}{*}{$\begin{array}{l}\vec{J} \\
\bar{z} \\
z \\
z\end{array}$} & \multirow[b]{2}{*}{ 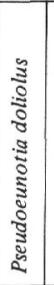 } & \multirow{2}{*}{\multicolumn{2}{|c|}{ 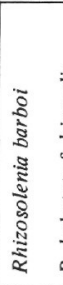 }} & \multirow[b]{2}{*}{ 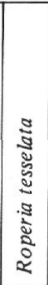 } & \multirow[b]{2}{*}{ 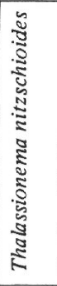 } & \multirow[b]{2}{*}{ 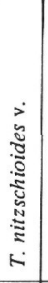 } & \multirow{2}{*}{\multicolumn{2}{|c|}{ 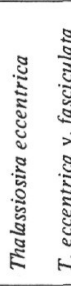 }} & & \multirow[b]{2}{*}{ 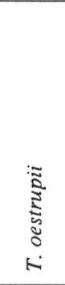 } & \multirow{2}{*}{\multicolumn{2}{|c|}{ 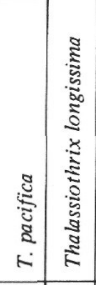 }} & \multirow[b]{2}{*}{ 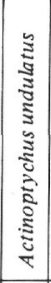 } & \multirow[b]{2}{*}{ 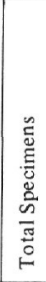 } & \multirow[b]{2}{*}{ 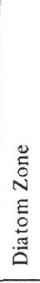 } & \multirow[b]{2}{*}{ 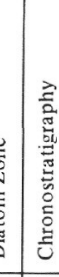 } \\
\hline 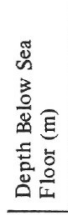 & 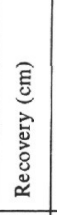 & 0 & 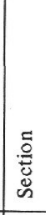 & 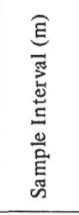 & 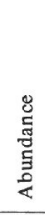 & 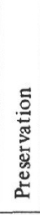 & & & & & & & & & & & & & & & & & & & & & & & & & & & & \\
\hline 5.5 & 3.0 & 1 & $\begin{array}{l}1 \\
2 \\
3 \\
4\end{array}$ & & $v_{K}$ & $\begin{array}{l}p \\
p \\
p\end{array}$ & $\begin{array}{l}1 \\
1 \\
2 \\
1\end{array}$ & $\begin{array}{l}7 \\
5 \\
5\end{array}$ & 2 & $\begin{array}{l}3 \\
1\end{array}$ & 1 & 1 & 1 & $\begin{array}{r}23 \\
21 \\
7\end{array}$ & 2 & 1 & $\begin{array}{l}1 \\
1\end{array}$ & $\begin{array}{l}1 \\
1\end{array}$ & $\begin{array}{r}3 \\
6 \\
10 \\
1\end{array}$ & 1 & 2 & $\begin{array}{l}2 \\
3 \\
2\end{array}$ & $\begin{array}{r}15 \\
15 \\
6\end{array}$ & 1 & 3 & 2 & 1 & $\begin{array}{l}1 \\
5 \\
4 \\
4\end{array}$ & & 2 & 2 & $\begin{array}{l}10 \\
87 \\
26\end{array}$ & 0 & $\bar{a}$ \\
\hline
\end{tabular}

currences of the marine planktonic species selected by Koizumi (1973a, 1975a, c) and Burckle (1972, manuscript) for the Neogene diatom zonation of the North Pacific Ocean. Additions to the regular counting are shown by a black circle in the distribution chart (Table 4).
Abundance is recorded as follows: $\mathrm{B}=$ barren; $\mathrm{VR}=$ very rare (less than 100 individuals on one slide, $18 \times 18$ $\mathrm{mm}$ ); $\mathrm{R}=$ rare (101 to 1000 individuals on one slide); $\mathrm{F}=$ few (1001 to 2000 individuals on one slide); $\mathrm{C}=$ common (2001 to 4000 individuals on one slide); and $\mathrm{A}=$ abundant (more than 4001 individuals on one slide). 
Preservation is recorded as follows: $\mathrm{p}=$ poor, $\mathrm{m}=$ moderate, and $\mathrm{g}=$ good, according to the degree of destruction of the diatom valves.

Diatom zonations follow those proposed by Koizumi (1975a, c) and Burckle (1972, manuscript). The age assignments for these diatom zones follow Koizumi (1977) and Burckle and Opdyke (1977).

In this paper, the Miocene/Pliocene boundary is placed at the top of Geomagnetic Epoch 5 (bottom of the Gilbert Reversed Magnetic Epoch), at an age of 5.10 m.y., and the Pliocene/Pleistocene boundary is placed at the bottom in the Olduvai Event of the Matuyama Reversed Epoch, at an age of about 1.8 m.y.

\section{SITE 430 \\ (lat $37^{\circ} 58.88 ' \mathrm{~N}$, long $170^{\circ} 35.45$ ' E, depth $1464 \mathrm{~m}$ )}

\section{Stratigraphic Setting}

Three holes were drilled on a lagoonal sediment pond of Ōjin Seamount (Figure 1). Hole 430 was abandoned because of caving, after recovering three cores of Quaternary to Eocene pebbly mudstone. Core 1 of Hole 430 had zero recovery. At Hole $430 \mathrm{~A}$, the drill penetrated 47.5 meters of Quaternary and upper Paleocene calcareous ooze and sand. A punch core at Hole 430B resulted in very poor recovery of Quaternary watery ooze.

\section{Diatoms and Age}

Diatoms are very few in two samples and absent in the core-catcher sample from Core 2 of Hole 430. Diatoms are present, but very rare, in only one sample from Core 1 of Hole 430A (Table 1).

Diatom-bearing samples of Site 430 belong to the Pseudoeunotia doliolus Zone (Recent to early Brunhes Normal Epoch; 0 to 0.63 m.y.B.P.) of Burckle (1972; in Burckle and Opdyke, 1977).

\section{SITE 431 \\ (lat $42^{\circ} 25.44^{\prime} \mathrm{N}$, long $170^{\circ} 32.68$ ' $\mathrm{E}$, depth $1714.5 \mathrm{~m}$ )}

\section{Stratigraphic Setting}

Two holes were drilled on a faulted terrace of Yōmei Seamount (Figure 1). At both Hole 431 and Hole 431A, drilling was ended after the recovery of only two cores, owing to the loss of the bottom hole assembly.

\section{Diatoms and Age}

Diatoms are very few in two samples from Core 1 of Hole 431 and in three samples from Core 1 of Hole 431A. They are completely absent in two samples from Core 2, Hole 431A (Table 2). No marine tychopelagic to benthic species or fresh-water species were observed in any of the samples. Diatom-bearing samples at Site 431 belong to the Denticula seminae Zone (Recent to late Brunhes Normal Epoch; 0 to 0.25 m.y.B.P.) of Koizumi (1973a, 1975a) and the Pseudoeunotia doliolus Zone.

\section{SITE 432 \\ (lat $41^{\circ} 20.03$ ' $\mathrm{N}$, long $170^{\circ} 74^{\prime} \mathrm{E}$, depth $1320 \mathrm{~m}$ )}

\section{Stratigraphic Setting}

Two holes were drilled on a terrace deposit atop Nintoku Seamount (Figure 1). At Hole 432, the lower bottom sub sheared off while we were attempting to take Core 2. Hole $432 \mathrm{~A}$ was washed down to a sub-bottom depth of 36 meters, and 3.52 meters (Cores 1 and 2) of well-consolidated Paleocene calcite-cemented conglomerate and sandstone, underlain by a thin red clay above basalt, was recovered.

\section{Diatoms and Age}

Diatoms are very few in four samples from Core 1 of Hole 432. The assemblages represent the Denticula seminae Zone and the Pseudoeunotia doliolus Zone (Table 3). All other samples are barren of diatoms.

\section{SITE 433 \\ (lat $44^{\circ} 46.60$ ' $\mathrm{N}$, long $170^{\circ} 01.26$ ' $\mathrm{E}$, depth $1874 \mathrm{~m}$ )}

\section{Stratigraphic Setting}

Four holes, including a multiple re-entry hole, were drilled at the southwestern flank of the central ridge of Suiko Seamount (Figure 1). At Hole 433, a 5.0-meter core of Pleistocene to upper Pliocene diatom-nannofossil ooze was taken. Hole 433A was continuously cored, and penetrated 163.5 meters of sedimentary rocks. The sedimentary section consists of five lithologic units: (1) Pleistocene to upper Pliocene foraminiferal nannofossil ooze from 0 to 5.0 meters (Core 1); (2) lower Pliocene through upper Miocene diatom-nannofossil ooze to marly siliceous nannofossil ooze from 5.0 to 43.0 meters (Cores 2 to 5); (3) upper Miocene calcareous ooze to calcareous chalk from 43.0 to 52.0 meters (Sections 1 to 6 of Core 6); (4) middle to lower Miocene tuffaceous sandy mud from 52.0 to 52.5 meters (Section 7 of Core 6); and (5) Paleocene carbonate sand and sandy mud from $\mathbf{5 2 . 5}$ to 163.5 meters (CC of Core 6 to Core 19). Hole 433B was washed to a depth of 128.5 meters sub-bottom, and cored in the lower $\mathbf{3 4 . 5}$ meters of sedimentary rocks of reef carbonate, sorted sandstone, and calcarenites ranging from lower Eocene to upper Paleocene. Hole $433 \mathrm{C}$ was washed to a depth of 163.0 meters sub-bottom and penetrated 4.5 meters of Paleocene calcarenite and reef carbonate sand, and 383.0 meters of basalt.

\section{Abundance and Preservation of Diatoms}

In general, common and moderately preserved diatoms are continuously present throughout the upper 52.3 meters of siliceous and calcareous ooze and calcareous chalk from Cores 1 to 6 of Hole 433A, except in the core-catcher sample of Core 6 which is barren of diatoms. Diatoms are poorly preserved in Cores 3 and 4, 
TABLE 4

Neogene Diatoms

\begin{tabular}{|c|c|c|c|c|c|c|c|c|c|c|c|c|c|c|c|c|c|c|c|c|c|c|c|c|}
\hline \multicolumn{8}{|c|}{ DSDP, Leg 55, Site 433} & \multirow[b]{2}{*}{ 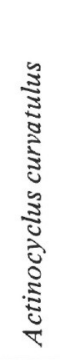 } & \multirow[b]{2}{*}{ 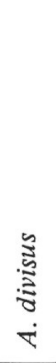 } & \multirow[b]{2}{*}{ 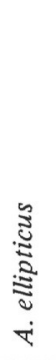 } & \multirow[b]{2}{*}{ 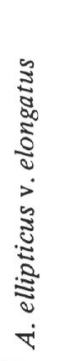 } & \multirow[b]{2}{*}{ 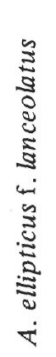 } & \multirow[b]{2}{*}{ 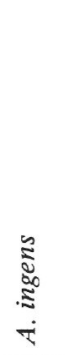 } & \multirow[b]{2}{*}{ 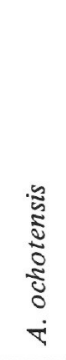 } & \multirow[b]{2}{*}{ 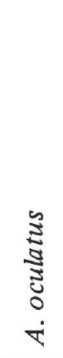 } & \multirow[b]{2}{*}{ 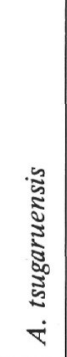 } & \multirow[b]{2}{*}{ 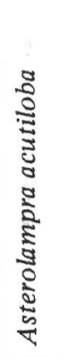 } & \multirow[b]{2}{*}{ 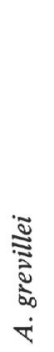 } & \multirow[b]{2}{*}{ 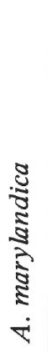 } & \multirow[b]{2}{*}{ 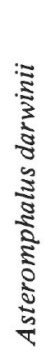 } & \multirow[b]{2}{*}{ 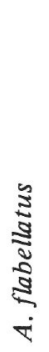 } & \multirow[b]{2}{*}{ 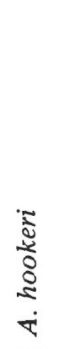 } & \multirow[b]{2}{*}{ 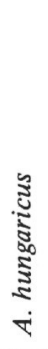 } & \multirow[b]{2}{*}{ 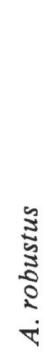 } \\
\hline $\begin{array}{l}\frac{0}{0} \\
\text { I }\end{array}$ & 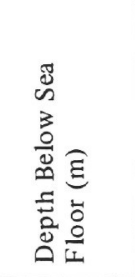 & 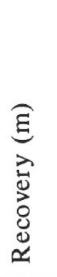 & 过 & 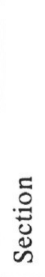 & 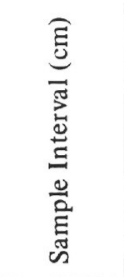 & 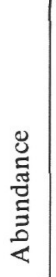 & 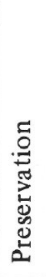 & & & & & & & & & & & & & & & & & \\
\hline & $0-5.0$ & 5.0 & 1 & $\begin{array}{l}1 \\
2 \\
3\end{array}$ & $\begin{array}{c}73-75 \\
8-10 \\
108-110 \\
90-92 \\
\text { CC }\end{array}$ & $\begin{array}{l}\mathrm{C} \\
\mathrm{R} \\
\mathrm{A} \\
\mathrm{A} \\
\mathrm{A}\end{array}$ & $\begin{array}{l}\mathrm{c} \\
\mathrm{c} \\
\mathrm{g} \\
\mathrm{g} \\
\mathrm{g}\end{array}$ & $\begin{array}{r}14 \\
10 \\
1 \\
2 \\
1\end{array}$ & $\begin{array}{l}1 \\
2\end{array}$ & 1 & & 1 & & $\begin{array}{r}\bullet \\
31 \\
1\end{array}$ & $\begin{array}{l}3 \\
1 \\
4 \\
1\end{array}$ & & & & & 1 & $\begin{array}{l}1 \\
1\end{array}$ & & & $\begin{array}{l}2 \\
3 \\
3\end{array}$ \\
\hline \multirow[t]{6}{*}{$A$} & $0-5.0$ & 1.7 & 1 & 1 & $\begin{array}{r}4-13 \\
18-20 \\
65-67 \\
\text { CC } \\
\end{array}$ & $\begin{array}{l}\mathrm{F} \\
\mathrm{R} \\
\mathrm{F} \\
\mathrm{F} \\
\end{array}$ & $\begin{array}{l}\mathrm{p} \\
\mathrm{p} \\
\mathrm{c} \\
\mathrm{c}\end{array}$ & $\begin{array}{l}7 \\
7 \\
2\end{array}$ & 1 & & & 1 & & $\begin{array}{r}2 \\
14 \\
2\end{array}$ & $\begin{array}{r}2 \\
3 \\
15 \\
2 \\
\end{array}$ & & & & & & & & & $\begin{array}{l}\bullet \\
1 \\
3 \\
\end{array}$ \\
\hline & $5.0-14.5$ & 2.4 & 2 & $\begin{array}{l}1 \\
2\end{array}$ & $\begin{array}{c}27-29 \\
120-122 \\
28-30 \\
\text { CC }\end{array}$ & $\begin{array}{l}\mathrm{F} \\
\mathrm{C} \\
\mathrm{C} \\
\mathrm{C}\end{array}$ & $\begin{array}{l}\mathrm{c} \\
\mathrm{c} \\
\mathrm{c} \\
\mathrm{c}\end{array}$ & $\begin{array}{l}1 \\
1 \\
5\end{array}$ & $\begin{array}{l}3 \\
1\end{array}$ & $\begin{array}{l}1 \\
2\end{array}$ & & & & $\begin{array}{r}3 \\
2 \\
2 \\
21\end{array}$ & 7 & & & & & $\begin{array}{l}\bullet \\
3\end{array}$ & $\bullet$ & $\begin{array}{l}1 \\
\bullet\end{array}$ & & $\begin{array}{l}\bullet \\
1 \\
2 \\
2\end{array}$ \\
\hline & $14.5-24.0$ & 8.0 & 3 & $\begin{array}{l}1 \\
2 \\
3 \\
4 \\
5 \\
6\end{array}$ & $\begin{array}{r}8-10 \\
8-10 \\
8-10 \\
18-20 \\
8-10 \\
8-10 \\
C C \\
\end{array}$ & $\begin{array}{l}A \\
A \\
C \\
F \\
F \\
F \\
C\end{array}$ & $\begin{array}{l}\mathrm{p} \\
\mathrm{p} \\
\mathrm{p} \\
\mathrm{p} \\
\mathrm{p} \\
\mathrm{p} \\
\mathrm{p}\end{array}$ & $\begin{array}{l}1 \\
1 \\
3 \\
2 \\
3 \\
\bullet \\
1\end{array}$ & 1 & $\begin{array}{l}1 \\
1 \\
2 \\
1\end{array}$ & • & $\begin{array}{l}1 \\
2 \\
1\end{array}$ & & $\begin{array}{l}- \\
1 \\
1 \\
2 \\
-\end{array}$ & & & & & $\begin{array}{l}2 \\
1 \\
- \\
\end{array}$ & $\begin{array}{l}4 \\
3 \\
2 \\
1 \\
1\end{array}$ & 1 & $\begin{array}{l}2 \\
2 \\
1\end{array}$ & & $\begin{array}{l}1 \\
1 \\
\\
1 \\
\bullet\end{array}$ \\
\hline & $24.0-33.5$ & 8.7 & 4 & $\begin{array}{l}1 \\
2 \\
3 \\
4 \\
5 \\
6\end{array}$ & $\begin{array}{l}8-10 \\
8-10 \\
8-10 \\
8-10 \\
8-10 \\
8-10 \\
C C\end{array}$ & $\begin{array}{l}\mathrm{C} \\
\mathrm{C} \\
\mathrm{F} \\
\mathrm{F} \\
\mathrm{C} \\
\mathrm{C} \\
\mathrm{A}\end{array}$ & $\begin{array}{l}\mathrm{p} \\
\mathrm{p} \\
\mathrm{p} \\
\mathrm{p} \\
\mathrm{c} \\
\mathrm{c} \\
\mathrm{c}\end{array}$ & $\begin{array}{l}2 \\
1 \\
1 \\
2 \\
1 \\
2 \\
1\end{array}$ & & $\begin{array}{l}1 \\
1 \\
\\
\\
2\end{array}$ & 2 & $\begin{array}{l}1 \\
1 \\
4 \\
1 \\
\\
1 \\
5\end{array}$ & & & & & $\begin{array}{l}2 \\
1 \\
1\end{array}$ & & $\begin{array}{l}1 \\
1 \\
2\end{array}$ & & & & $\bullet$ & \\
\hline & $33.5-43.0$ & 8.6 & 5 & $\begin{array}{l}1 \\
2 \\
3 \\
4 \\
5 \\
6\end{array}$ & $\begin{array}{l}8-10 \\
8-10 \\
8-10 \\
8-10 \\
8-10 \\
8-10 \\
\text { CC }\end{array}$ & $\begin{array}{l}\mathrm{F} \\
\mathrm{F} \\
\mathrm{C} \\
\mathrm{F} \\
\mathrm{A} \\
\mathrm{A} \\
\mathrm{R}\end{array}$ & $\begin{array}{l}\mathrm{c} \\
\mathrm{c} \\
\mathrm{c} \\
\mathrm{c} \\
\mathrm{g} \\
\mathrm{g} \\
\mathrm{c}\end{array}$ & $\begin{array}{l}4 \\
1 \\
1 \\
2 \\
1 \\
1\end{array}$ & & $\begin{array}{l}3 \\
2 \\
\\
\\
\\
1\end{array}$ & 1 & $\begin{array}{l}1 \\
1\end{array}$ & & & & & - & $\begin{array}{l}1 \\
1\end{array}$ & $\bullet$ & $\begin{array}{l}2 \\
\bullet \\
1\end{array}$ & & 1 & $\begin{array}{l}\bullet \\
\dot{\bullet} \\
1 \\
1\end{array}$ & \\
\hline & $43.0-52.5$ & 9.5 & 6 & $\begin{array}{l}1 \\
2 \\
3 \\
4 \\
5 \\
6\end{array}$ & $\begin{array}{c}8-10 \\
8-10 \\
8-10 \\
8-10 \\
8-10 \\
8-10 \\
53-54 \\
114-115 \\
138-140 \\
6-7 \\
27-29\end{array}$ & $\begin{array}{l}\text { A } \\
\text { C } \\
\text { C } \\
\text { C } \\
\text { C } \\
\text { C } \\
\text { C } \\
\text { F } \\
\text { F } \\
\text { F } \\
\text { R }\end{array}$ & $\begin{array}{l}\mathrm{g} \\
\mathrm{g} \\
\mathrm{g} \\
\mathrm{g} \\
\mathrm{g} \\
\mathrm{g} \\
\mathrm{g} \\
\mathrm{p} \\
\mathrm{p} \\
\mathrm{p} \\
\mathrm{p}\end{array}$ & $\begin{array}{l}1 \\
2 \\
1 \\
1\end{array}$ & & $\begin{array}{l}1 \\
1 \\
1 \\
3\end{array}$ & & & $\begin{array}{l}45 \\
29 \\
37 \\
30\end{array}$ & & & $\begin{array}{r}100 \\
68 \\
49 \\
132\end{array}$ & $\begin{array}{l}2 \\
1 \\
2 \\
- \\
\end{array}$ & 1 & \begin{tabular}{l|} 
\\
$\bullet$ \\
1 \\
$\bullet$
\end{tabular} & 2 & & & $\begin{array}{l}1 \\
1 \\
1 \\
1 \\
1 \\
2 \\
1\end{array}$ & \\
\hline
\end{tabular}

and they are rare to few and badly preserved in the lowermost part of Core $6(51.64$ to $52.3 \mathrm{~m})$. All samples taken from below Core 7 of Hole 433A and from Hole 433B are barren of diatoms.

Marine tychopelagic species occur frequently throughout the upper Miocene section of Core 6 (Table 4, Figure 2).

\section{Age, Biostratigraphy, and Paleoceanography}

Five diatom zones were recognized with zonal breaks (Table 5). The Denticula seminae Zone assemblage in a Sample 1-1, 73-75 cm is followed by the Rhizosolenia curvirostris Zone assemblage in Sample 1-2, 8-10 cm of Hole 433. After the absence of two zonal assemblages, 


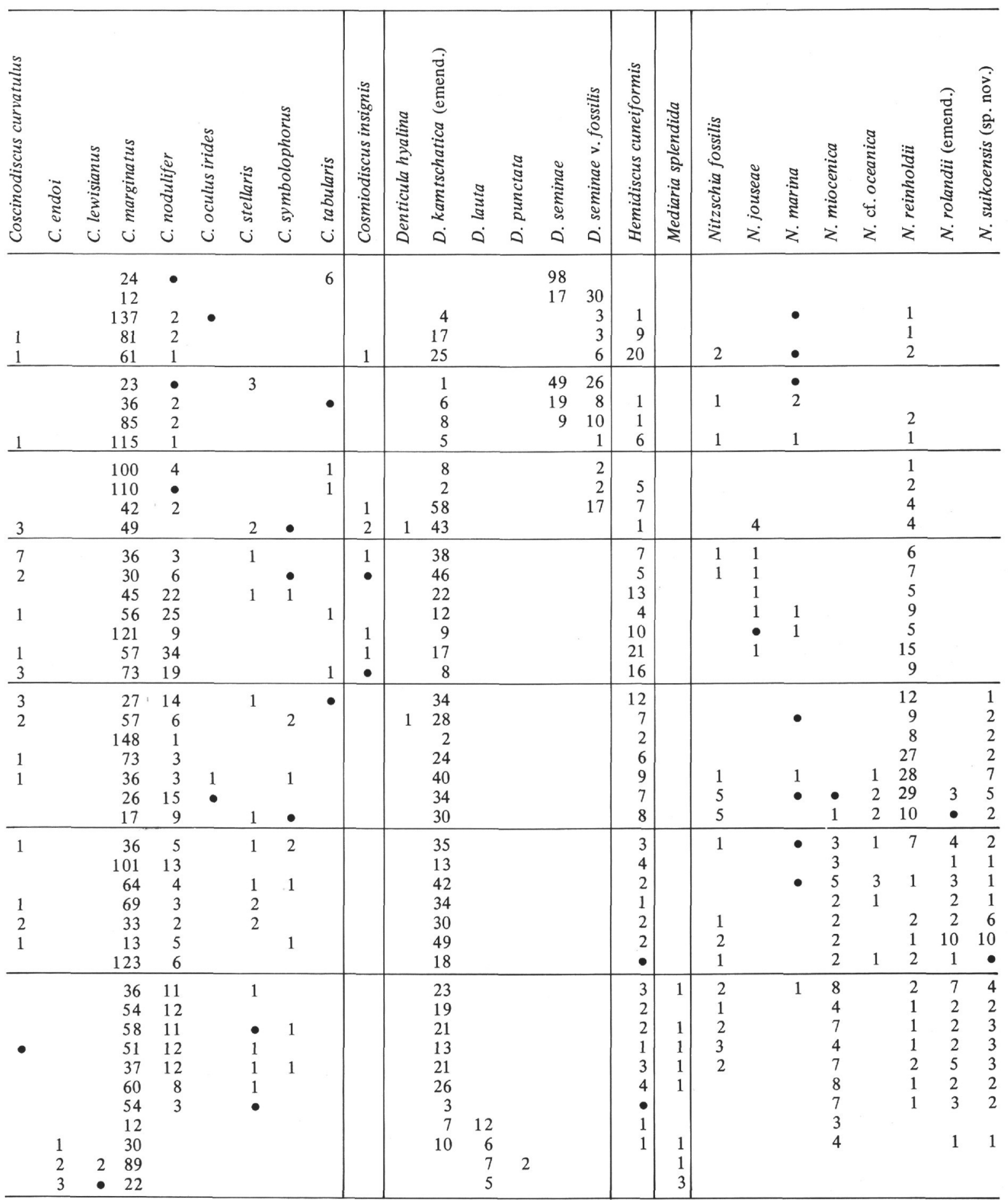

those of the Actinocyclus oculatus Zone and the Denticula seminae var. fossilis Zone, the Denticula seminae var. fossilis-Denticula kamtschatica Zone assemblage was observed in Samples 1-2, 108-110 cm to 3,CC. In Hole 433A, the uppermost Denticula seminae Zone assemblage was not found, owing to a lack of sediments representing this zone. The same two zones that are missing in Hole 433 are also absent between Samples $1-1,18-20 \mathrm{~cm}$ and 1-1, 65-67 cm. The Denticula kam- tschatica Zone assemblage occurs in the interval between Samples 2,CC and 6-6, 53-54 cm. Older reworked middle Miocene species from the Denticula lauta Zone assemblage are common in two samples, 6-6, 114-115 $\mathrm{cm}$ and $6-6,138-140 \mathrm{~cm}$, which originally belonged to the Denticula kamtschatica Zone. This interval, therefore, is the mixed zone of both Denticula kamtschatica Zone and Denticula lauta Zone assemblages. Below the mixed interval, the Denticula lauta Zone assemblage 
TABLE 4 - Continued

\begin{tabular}{|c|c|c|c|c|c|c|c|c|c|c|c|c|c|c|c|c|c|c|c|c|c|c|c|c|}
\hline \multicolumn{8}{|c|}{ DSDP, Leg 55, Site 433} & \multirow[b]{2}{*}{ 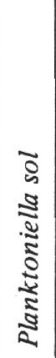 } & \multirow[b]{2}{*}{ 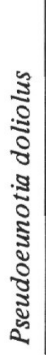 } & \multirow[b]{2}{*}{ 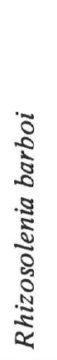 } & \multirow[b]{2}{*}{ 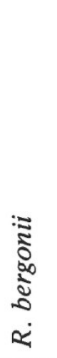 } & \multirow[b]{2}{*}{ 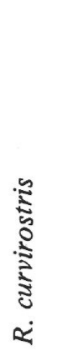 } & \multirow[b]{2}{*}{ 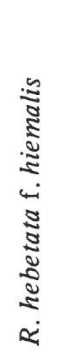 } & \multirow[b]{2}{*}{ 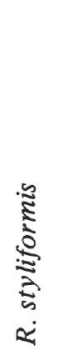 } & \multirow[b]{2}{*}{$\begin{array}{l}\infty \\
\dot{2} \\
\dot{2} \\
\dot{1}\end{array}$} & \multirow[b]{2}{*}{$\begin{array}{l}\dot{0} \\
\dot{8} \\
\dot{\alpha}\end{array}$} & \multirow[b]{2}{*}{$\begin{array}{l}4 \\
\dot{2} \\
\dot{2} \\
\dot{2}\end{array}$} & \multirow[b]{2}{*}{ 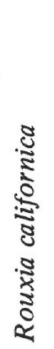 } & \multirow[b]{2}{*}{ 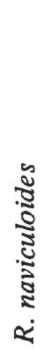 } & \multirow[b]{2}{*}{ 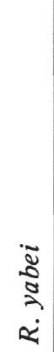 } & \multirow[b]{2}{*}{ 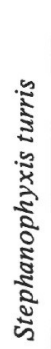 } & \multirow[b]{2}{*}{ 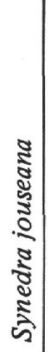 } & \multirow[b]{2}{*}{ 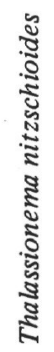 } & \multirow[b]{2}{*}{ 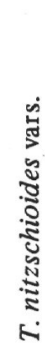 } \\
\hline$\frac{0}{0}$ & 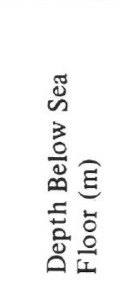 & 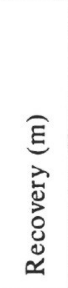 & نั & 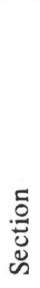 & 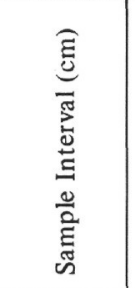 & 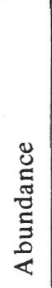 & 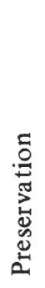 & & & & & & & & & & & & & & & & & \\
\hline & $0-5.0$ & 5.0 & 1 & $\begin{array}{l}1 \\
2\end{array}$ & $\begin{array}{c}73-75 \\
8-10 \\
108-110 \\
90-92 \\
\mathrm{CC} \\
\end{array}$ & \begin{tabular}{l|}
$\mathrm{C}$ \\
$\mathrm{R}$ \\
$\mathrm{A}$ \\
$\mathrm{A}$ \\
$\mathrm{A}$ \\
\end{tabular} & $\begin{array}{l}\mathrm{c} \\
\mathrm{c} \\
\mathrm{g} \\
\mathrm{g} \\
\mathrm{g}\end{array}$ & & $\bullet$ & $\begin{array}{r}15 \\
5 \\
7 \\
6 \\
\end{array}$ & $\begin{array}{l}2 \\
1\end{array}$ & 20 & $\begin{array}{r}15 \\
11 \\
2 \\
1 \\
\bullet\end{array}$ & • & & 1 & & & & & $\begin{array}{l}3 \\
\bullet \\
1 \\
2\end{array}$ & & $\begin{array}{r}3 \\
7 \\
5 \\
19 \\
18 \\
\end{array}$ & $\begin{array}{l}3 \\
\dot{1} \\
2 \\
\end{array}$ \\
\hline \multirow[t]{6}{*}{ A } & $0-5.0$ & 1.7 & 1 & 1 & $\begin{array}{r}4-13 \\
18-20 \\
65-67 \\
\text { CC }\end{array}$ & $\begin{array}{l}\mathrm{F} \\
\mathrm{R} \\
\mathrm{F} \\
\mathrm{F}\end{array}$ & $\begin{array}{l}\mathrm{p} \\
\mathrm{p} \\
\mathrm{c} \\
\mathrm{c}\end{array}$ & & $\dot{3}$ & $\begin{array}{r}1 \\
14 \\
12 \\
7\end{array}$ & & $\begin{array}{r}6 \\
19 \\
\bullet\end{array}$ & $\begin{array}{r}15 \\
6\end{array}$ & 6 & & & & & & & $\begin{array}{l}4 \\
1 \\
\bullet \\
2\end{array}$ & & $\begin{array}{r}8 \\
13 \\
9 \\
6\end{array}$ & $\begin{array}{l}4 \\
2 \\
1\end{array}$ \\
\hline & $5.0-14.5$ & 2.4 & 2 & 1 & $\begin{array}{c}27-29 \\
120-122 \\
28-30 \\
\mathrm{CC} \\
\end{array}$ & $\begin{array}{l}\mathrm{F} \\
\mathrm{C} \\
\mathrm{C} \\
\mathrm{C} \\
\end{array}$ & $\begin{array}{l}\mathrm{c} \\
\mathrm{c} \\
\mathrm{c} \\
\mathrm{c}\end{array}$ & & & $\begin{array}{l}5 \\
6 \\
3 \\
4 \\
\end{array}$ & 1 & & $\begin{array}{l}3 \\
2 \\
1 \\
1 \\
\end{array}$ & & 1 & & & & & & $\begin{array}{l}4 \\
2 \\
3 \\
3 \\
\end{array}$ & & $\begin{array}{r}2 \\
7 \\
15 \\
28 \\
\end{array}$ & $\begin{array}{l}1 \\
4 \\
- \\
\end{array}$ \\
\hline & $14.5-24.0$ & 8.0 & 3 & $\begin{array}{l}1 \\
2 \\
3 \\
4 \\
5 \\
6\end{array}$ & $\begin{array}{r}8-10 \\
8-10 \\
8-10 \\
18-20 \\
8-10 \\
8-10 \\
\text { CC }\end{array}$ & $\begin{array}{l}\text { A } \\
\text { A } \\
\text { C } \\
\text { F } \\
\text { F } \\
\text { F } \\
\text { C }\end{array}$ & $\begin{array}{l}\mathrm{p} \\
\mathrm{p} \\
\mathrm{p} \\
\mathrm{p} \\
\mathrm{p} \\
\mathrm{p} \\
\mathrm{p}\end{array}$ & & & $\begin{array}{l}1 \\
\bullet \\
1 \\
3 \\
2 \\
2 \\
1\end{array}$ & $\begin{array}{l}3 \\
6 \\
\bullet \\
4 \\
5 \\
8 \\
5\end{array}$ & & $\begin{array}{l}1 \\
\bullet \\
- \\
2\end{array}$ & & & & 1 & & & & $\begin{array}{l}1 \\
\bullet \\
2 \\
2 \\
2\end{array}$ & & $\begin{array}{r}32 \\
25 \\
32 \\
33 \\
8 \\
18 \\
31\end{array}$ & $\begin{array}{l}4 \\
3\end{array}$ \\
\hline & $24.0-33.5$ & 8.7 & 4 & $\begin{array}{l}1 \\
2 \\
3 \\
4 \\
5 \\
6\end{array}$ & $\begin{array}{l}8-10 \\
8-10 \\
8-10 \\
8-10 \\
8-10 \\
8-10 \\
\text { CC }\end{array}$ & $\begin{array}{l}\mathrm{C} \\
\mathrm{C} \\
\mathrm{F} \\
\mathrm{F} \\
\mathrm{C} \\
\mathrm{C} \\
\mathrm{A}\end{array}$ & $\begin{array}{l}\mathrm{p} \\
\mathrm{p} \\
\mathrm{p} \\
\mathrm{p} \\
\mathrm{p} \\
\mathrm{c} \\
\mathrm{c}\end{array}$ & $\begin{array}{l}1 \\
1\end{array}$ & & $\begin{array}{l}1 \\
2\end{array}$ & & & $\begin{array}{l}3 \\
1 \\
2 \\
1 \\
2 \\
2 \\
4\end{array}$ & $\begin{array}{l}1 \\
1 \\
\\
2 \\
2 \\
\end{array}$ & $\begin{array}{r}12 \\
6 \\
1 \\
15 \\
14 \\
10 \\
16 \\
\end{array}$ & 3 & $\begin{array}{l}6 \\
5 \\
\bullet \\
2 \\
1 \\
4 \\
6\end{array}$ & $\begin{array}{r}15 \\
8 \\
1 \\
7 \\
12 \\
8 \\
22 \\
\end{array}$ & & $\begin{array}{l}2 \\
1 \\
\end{array}$ & & & $\begin{array}{l}24 \\
21 \\
11 \\
12 \\
14 \\
11 \\
25 \\
\end{array}$ & $\begin{array}{l}2 \\
2 \\
6 \\
6\end{array}$ \\
\hline & $33.5-43.0$ & 8.6 & 5 & $\begin{array}{l}1 \\
2 \\
3 \\
4 \\
5 \\
6\end{array}$ & $\begin{array}{l}8-10 \\
8-10 \\
8-10 \\
8-10 \\
8-10 \\
8-10 \\
\text { CC }\end{array}$ & $\begin{array}{l}\mathrm{F} \\
\mathrm{F} \\
\mathrm{C} \\
\mathrm{F} \\
\mathrm{A} \\
\mathrm{A} \\
\mathrm{R}\end{array}$ & $\begin{array}{l}\mathrm{c} \\
\mathrm{c} \\
\mathrm{c} \\
\mathrm{c} \\
\mathrm{g} \\
\mathrm{g} \\
\mathrm{c}\end{array}$ & 1 & & $\begin{array}{r}2 \\
\bullet \\
5 \\
5 \\
10 \\
12 \\
4\end{array}$ & & & $\begin{array}{r}7 \\
6 \\
3 \\
9 \\
5 \\
4 \\
11\end{array}$ & 2 & $\begin{array}{r}11 \\
5 \\
6 \\
15 \\
9 \\
3 \\
5\end{array}$ & 2 & $\begin{array}{l}4 \\
2 \\
- \\
3 \\
6 \\
2 \\
\bullet\end{array}$ & $\begin{array}{r}8 \\
7 \\
8 \\
5 \\
11 \\
6 \\
\bullet\end{array}$ & & $\begin{array}{l}2 \\
\dot{1} \\
1 \\
1 \\
\\
3\end{array}$ & $\begin{array}{l}2 \\
3 \\
2 \\
\bullet \\
3 \\
\\
1\end{array}$ & $\begin{array}{l}3 \\
2 \\
1\end{array}$ & $\begin{array}{l}15 \\
10 \\
17 \\
16 \\
37 \\
57 \\
11\end{array}$ & $\begin{array}{l}4 \\
2 \\
1 \\
8 \\
8 \\
6 \\
1\end{array}$ \\
\hline & $43.0-52.5$ & 9.5 & 6 & $\begin{array}{l}1 \\
2 \\
3 \\
4 \\
5 \\
6\end{array}$ & $\begin{array}{c}8-10 \\
8-10 \\
8-10 \\
8-10 \\
8-10 \\
8-10 \\
53-54 \\
114-115 \\
138-140 \\
6-7 \\
27-29\end{array}$ & $\begin{array}{l}\text { A } \\
\text { C } \\
\text { C } \\
\text { C } \\
\text { C } \\
\text { C } \\
\text { C } \\
\text { F } \\
\text { F } \\
\text { F } \\
\text { R }\end{array}$ & $\begin{array}{l}\mathrm{g} \\
\mathrm{g} \\
\mathrm{g} \\
\mathrm{g} \\
\mathrm{g} \\
\mathrm{g} \\
\mathrm{g} \\
\mathrm{p} \\
\mathrm{p} \\
\mathrm{p} \\
\mathrm{p}\end{array}$ & - & & & & & $\begin{array}{r} \\
8 \\
8 \\
5 \\
7 \\
7 \\
15\end{array}$ & $\begin{array}{l}2 \\
3 \\
\bullet \\
2 \\
2 \\
1\end{array}$ & $\begin{array}{l}2 \\
4 \\
2 \\
5 \\
5 \\
4 \\
8 \\
1 \\
5\end{array}$ & & $\begin{array}{l}3 \\
2 \\
3 \\
5 \\
1 \\
2\end{array}$ & $\begin{array}{r}10 \\
4 \\
3 \\
3 \\
1 \\
3 \\
3 \\
- \\
2\end{array}$ & $\begin{array}{l}1 \\
1\end{array}$ & $\begin{array}{l}1 \\
1 \\
1 \\
1\end{array}$ & $\begin{array}{l}2 \\
2 \\
1\end{array}$ & $\begin{array}{l}2 \\
3 \\
1 \\
2 \\
\bullet \\
3 \\
5 \\
2\end{array}$ & $\begin{array}{r}22 \\
23 \\
22 \\
22 \\
22 \\
21 \\
49 \\
10 \\
15 \\
1 \\
1\end{array}$ & $\begin{array}{l}2 \\
2 \\
1 \\
2\end{array}$ \\
\hline
\end{tabular}

was recognized in the interval between Samples 6-7, 6-7 $\mathrm{cm}$ and 6-7, 27-29 $\mathrm{cm}$, without two zonal assemblages, the Denticula hustedtii Zone and Denticula hustedtiiDenticula lauta Zone assemblages.

Figure 3 represents the ranges of selected marine planktonic diatom species and the boundaries between diatom zones, corresponding to depth below the sediment surface at Site 433 . The occurrences of a number of species have been directly compared with the paleomagnetic time-scale (Burckle, 1972, manuscript; Bur- ckle and Opdyke, 1977) and various radiometric dates (Koizumi, 1977). These absolute ages of diatom events were used to construct the curve of sediment accumulation rates at Site 433 (Figure 4).

Three hiatuses are shown in Figure 4. The Pliocene/Pleistocene hiatus is represented in Core 1 of Hole 433 and Core 1 of Hole 433A. The time gap over the boundary is calculated at about $1.7 \mathrm{~m} . \mathrm{y}$. This hiatus is marked by occurrence of sandy diatom-nannofossil ooze at a sub-bottom depth of from 1.30 to 2.15 meters. 
TABLE 4 - Continued

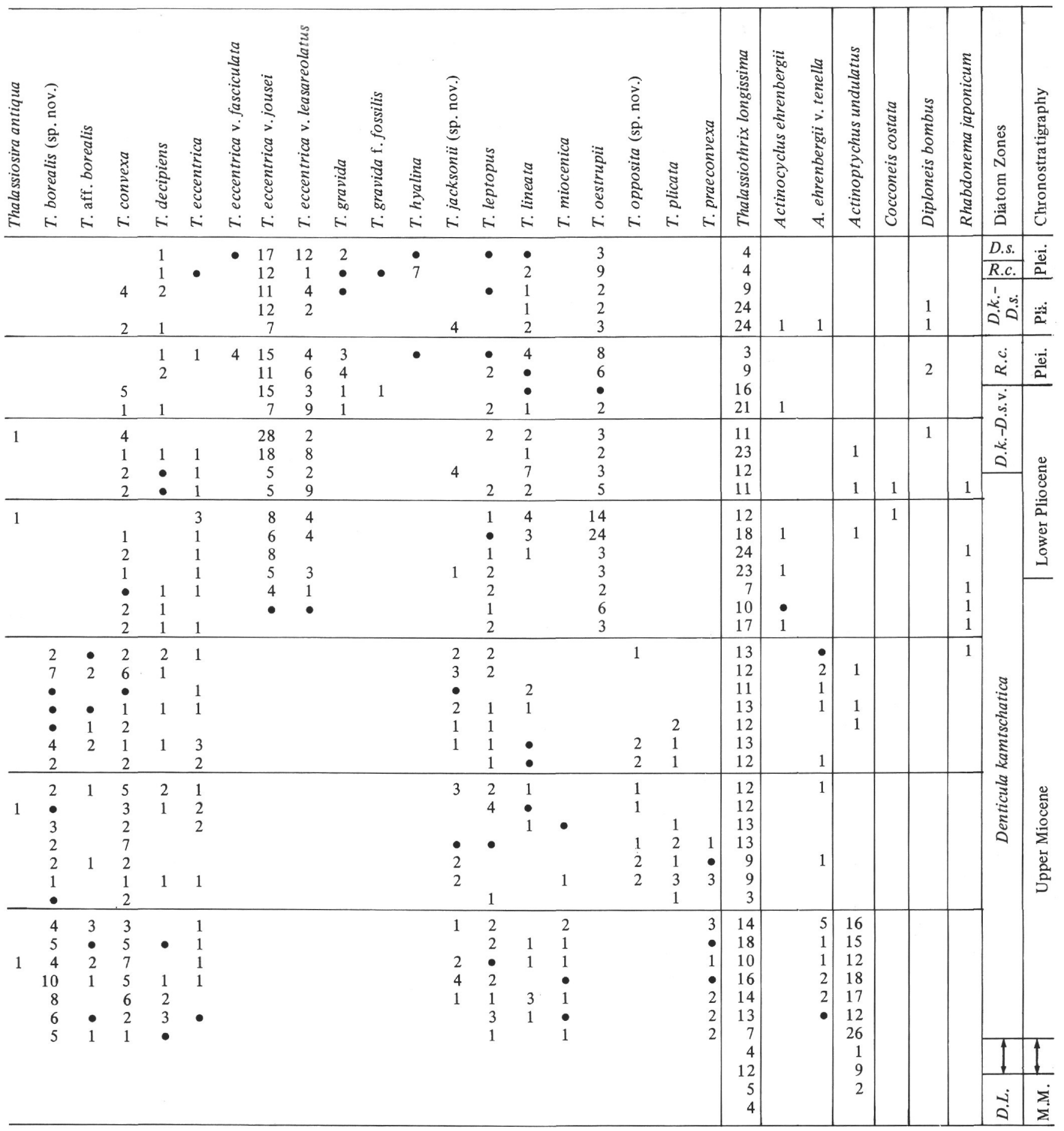

The boundary unconformity could be attributed to subbottom erosion of the top of the seamount by cold bottom currents. The Miocene/Pliocene hiatus was also identified between Cores 3 and 4 , where its duration was about $0.8 \mathrm{~m} . \mathrm{y}$. Another gap in Core 6 spans an interval of about 9.0 m.y., encompassing most of the late Miocene and the whole middle Miocene. The hiatus is also marked by occurrences of calcareous chalk and marly siliceous ooze at a sub-bottom depth of from 52.0 to 52.5 meters. These unconformities are assumed to have been caused by increased bottom water activity associated with global cooling in the latest Miocene to earliest Pliocene.

An abrupt change in sedimentation occurred about $4.4 \mathrm{~m} . \mathrm{y}$. ago. The accumulation rate for the upper 4.6 meters of Pliocene sediment was about 4.3 meters/m.y., whereas the estimated rate of accumulation for the lowermost Pliocene section between 6.8 and 23.8 meters was 53.0 meters $/ \mathrm{m} . y$. The sediments were deposited, therefore, about 12 times faster in the earliest Pliocene 


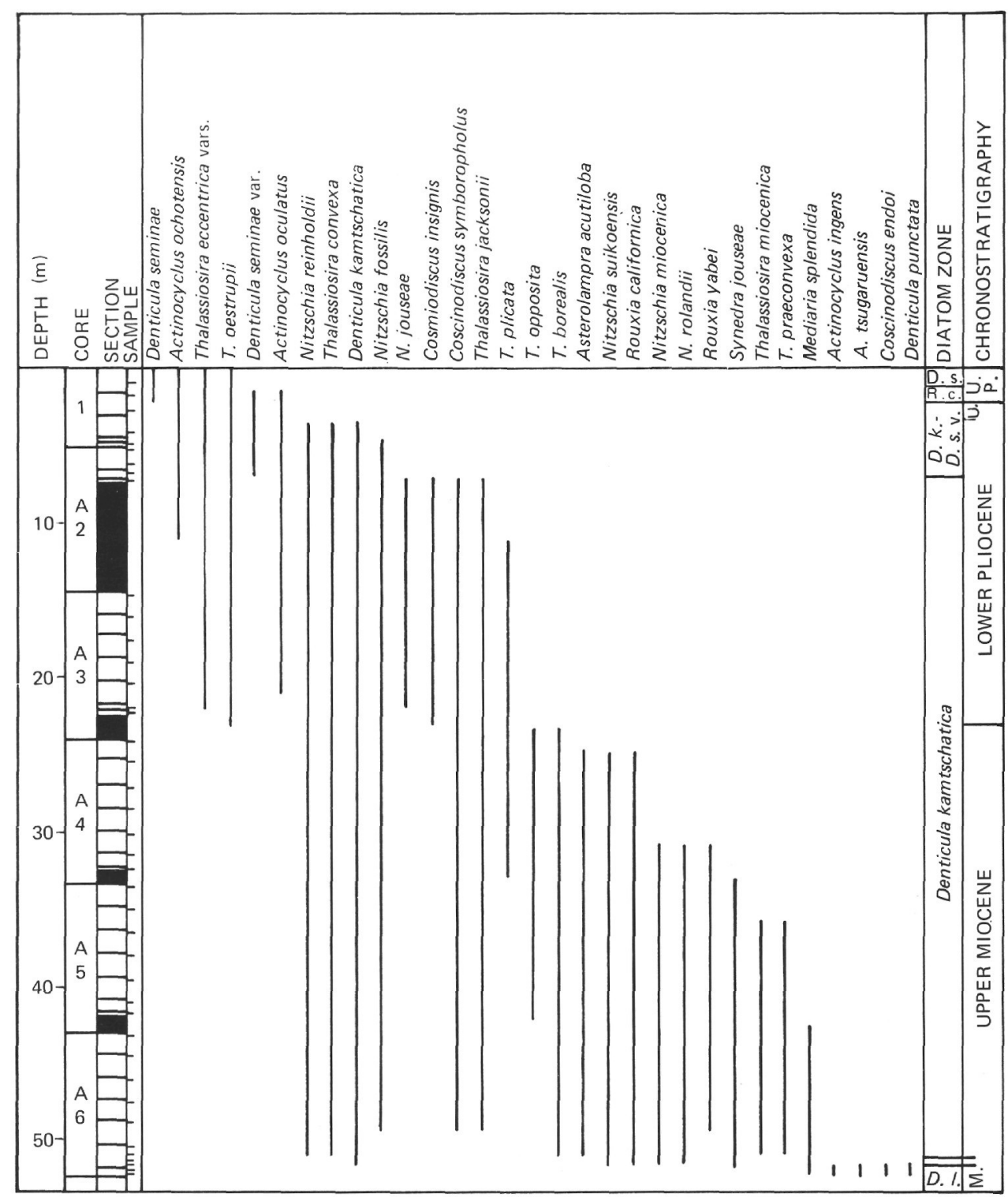

Figure 2. Chronostratigraphic positions of samples, based on accumulation rates (Figure 4), and paleoceanographic cycles at Site 433.

than during the later Pliocene. The calculated rate of deposition for the uppermost Miocene sediments is 30.0 meters/m.y., which is comparable to the lowest Pliocene rates.

$T d$ (diatom temperature) values (Kanaya and Koizumi, 1966) were calculated for each sample on the basis of the species assigned to cold or warm water (Figure 2). They are shown as $T d^{\prime}$ because the calculation involved extinct species. The abrupt increases in the diatom temperature curve are found both below and above the Miocene/Pliocene boundary. The trend of the curve is not shown near the boundary, because of the absence of samples due to the hiatus. The significant cooling, however, is recorded in the uppermost Miocene sections throughout the North Pacific region.

\section{DESCRIPTIONS OF FOUR NEW DIATOM SPECIES Genus NITZSCHIA Hassal (1845) \\ Nitzschia suikoensis Koizumi, n. sp. (Plate 1, Figures 1-6)}

Description: Valves linear-elliptic to elliptic with bluntly rounded apices, 25-60 $\mu$ long, 6-7 $\mu$ wide. Transapical striae straight in the middle of the valves, curved near the apices, $12-15$ in $10 \mu$, punctation not visible in the normal light microscope. Keel very eccentric, fibulae 6-8 in $10 \mu$, without a central nodule.

Holotype: Plate 1, Figures 3 and 4, $41 \mu$ in length, from DSDP Leg 55, Sample 443A-5-5, 8-10 cm, North Pacific Ocean.

Paratype: Plate 1, Figures 1 and 2, $30 \mu$ in length, from DSDP Leg 55, Sample 443A-6-6, 8-10 cm, North Pacific Ocean.

Discussion: No similar species were found in the literature. The new species is named for Suiko Seamount in the Emperor Seamount chain. 
TABLE 5

Neogene Diatoms

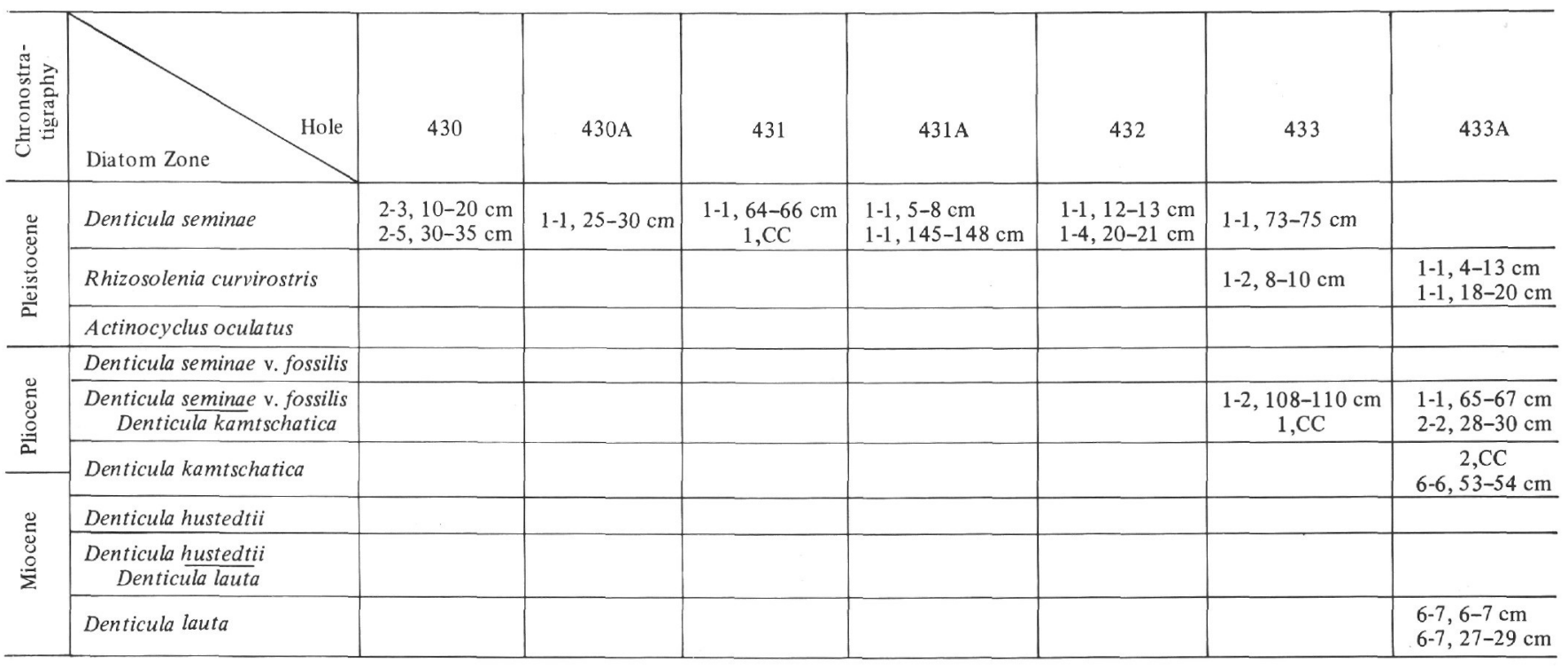

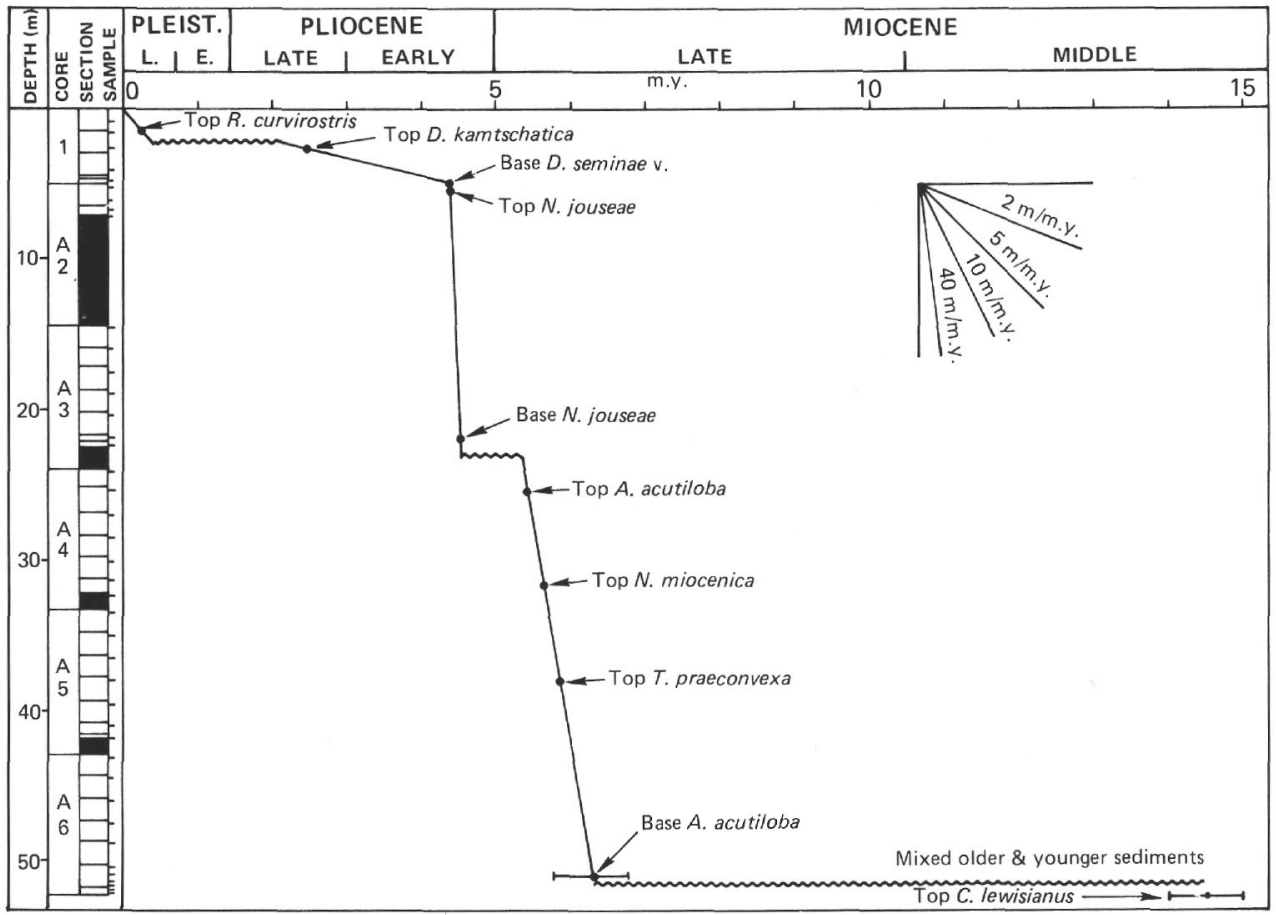

Figure 3. Stratigraphic ranges of selected marine planktonic diatom species and zonal subdivisions at Site 443. Black area in the section column shows unrecovered interval.

Occurrences: Up to now found only in the upper Miocene sediment from DSDP Hole 433A.

Genus THALASSIOSIRA Cleve (1873) emend. Hasle (1973)

Thalassiosira borealis Koizumi, n. sp.

(Plate 1, Figures 7-10)

Synonyms: Thalassiosira decipiens (Grun.) Jörg.?, SheshukovaPoretzkaya, 1967, pl. 4, fig. 5, pl. 14, fig. 2; Thalassiosira decipiens (?) Jörg., Schrader, 1973a, pl. 16, fig. 12; Thalassiosira nativa Sheshuk., Koizumi, 1975a, pl. 4, fig. 21, 22.

Description: Valves circular, $15-25 \mu$ in diameter, slightly curved, with rounded margins. Some small mucilage pores present in the depressed center. Areolae hexagonal, decreasing in size from the center to the margin, 8-12 in $10 \mu$ midway. Areolae arranged in slightly curved tangential rows with secondary sculpture of outwardly concave rows in outer border. One row of conspicuous marginal processes surround the inner edge of margin, about 4 in $10 \mu$.

Holotype: Plate 1, Figure 7, $17 \mu$ in diameter, from DSDP Leg 55, Sample 443A-6-3, 8-10 cm, North Pacific Ocean.

Paratype: Plate 1, Figure 8, $21 \mu$ in diameter, from DSDP Leg 55, Sample 433A-5-5, 8-10 cm, North Pacific Ocean.

Discussion: This species is similar to Thalassiosira decipiens (Grun.) Jörg., but differs by the presence of some mucilage pores in the center and of conspicuous marginal processes, and to Thalassiosira burcklina Schrader, 1974, p. 916, pl. 1, fig. 21-26, from which 


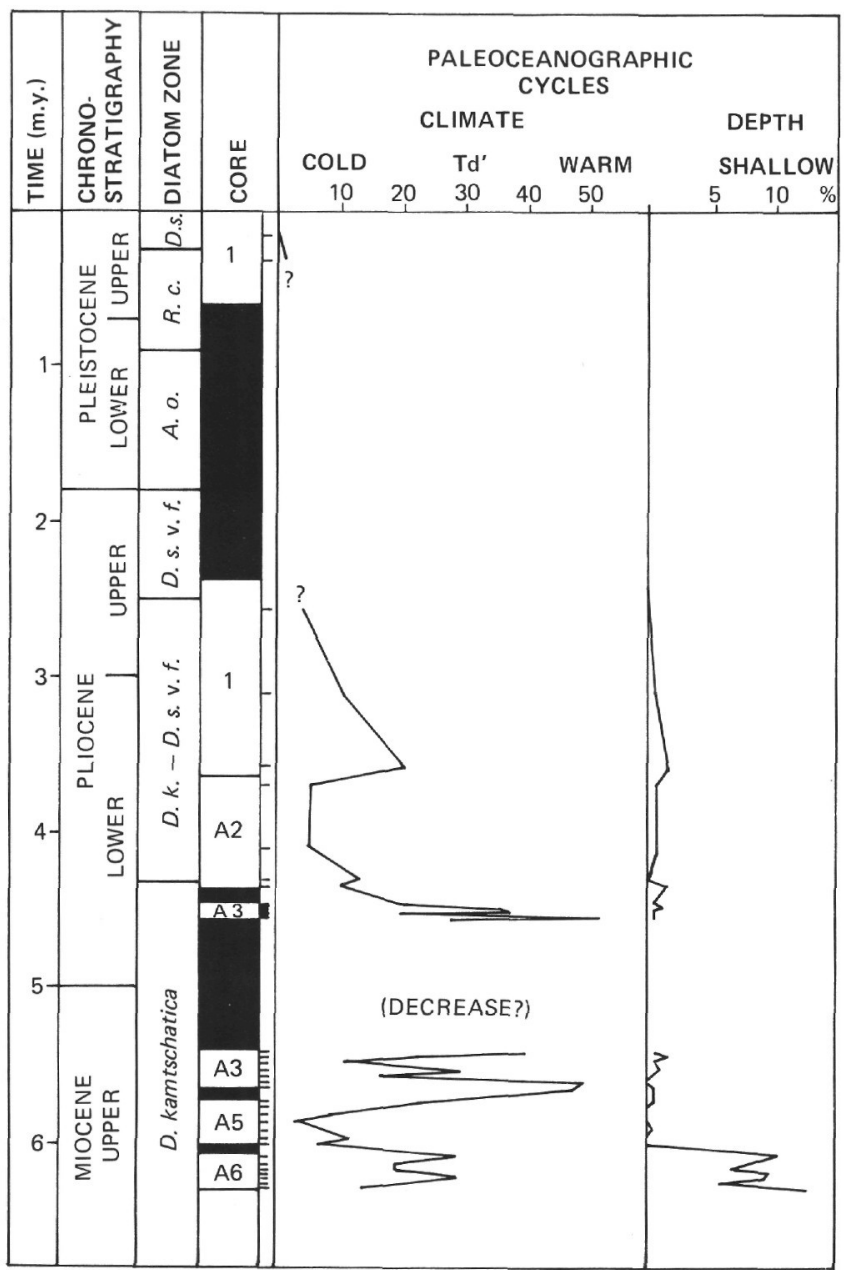

Figure 4. Accumulation rates of Neogene sediments at Site 433. Black area in the section column shows unrecovered interval.

it differs by the finer areolation, the arrangement of areolae, and the presence of marginal processes. This species is also distinguished from Thalassiosira nativa Sheshukova-Poretzkaya, 1967, p. 145, pl. 14, fig. $7 \mathrm{a}-\mathrm{B}$, by the larger form and the presence of marginal processes.

Occurrences: Thalassiosira borealis occurs in upper Miocene to lower Pliocene sediments from DSDP Sites 173, 302, 433, and land sections of Sakhalin and Kamchatka.

Thalassiosira jacksonii Koizumi and Barron, n. sp. (Plate 1, Figures 11-14)

Synonyms: Coscinodiscus symbolophorus Grun., SheshukovaPoretzkaya, 1967, pl. 1, fig. 3, pl. 22, fig. 3; Thalassiosira antarctica Comber, Schrader, 1974, pl. 3, fig. 10-13; Thalassiosira sp. b, Schrader and Fenner, 1976, pl. 17, fig. 5, 10.

Description: Valves circular, slightly convex, depressed in the center, with narrow margin. Diameter 13-30 $\mu$. Areolae circular, arranged in radial fascicles, within each fascicle parallel to the longest radial row. Areolae are also arranged weakly concave outward near the margin. Areolae slightly large and somewhat sparse in the center, smaller and denser outward, 13-18 in $10 \mu$ midway. A mucilage pore present in the hyaline central area. One row of marginal processes surrounds the inner edge of margin, 6-7 in $10 \mu$. All other rows of marginal spines to striae were barely distinguishable in the normal light microscope.

Holotype: Plate 1, Figure 12, $16 \mu$ in diameter, from DSDP Leg 55, Sample 433A-4-5, 8-10 cm, North Pacific Ocean.

Paratype: Plate 1, Figure 14, $20 \mu$ in diameter, from DSDP Leg 55, Sample 443A-1, CC, North Pacific Ocean.
Discussion: This species differs, by the presence of a mucilage pore in the hyaline center, from Thalassiosira antarctica Comber, 1896: Hendey, 1937, p. 237; Hustedt, 1958, p. 108, pl. 3, fig. 1-3; Hasle and Heimdal, 1968 , p. 357 , fig $1-21$, and by the areolation in radial to bifurcate rows and by the flat to slightly convex valve surface from Thalassiosira gracilis (Karst.) Hustedt, 1958: p. 109, fig. 4-7; Fenner, Schrader and Wienigk, 1976, p. 780, pl. 9, fig. 12-20. The new species is named for the late Dr. Dale E. Jackson, former senior researcher in marine geology, U.S. Geological Survey at Menlo Park, who served as co-chief scientist of DSDP Leg 55.

Occurrences: Thalassiosira jacksonii occurs in the Pliocene to upper Miocene sediments from DSDP Sites 238, 348, 433, 438, and land sections of Hokkaido and Sakhalin.

Thalassiosira opposita Koizumi, n. sp. (Plate 1, Figures 15-17)

Description: Valves flat to slightly curved, depressed in the center, with rounded margin. Diameter of valves $17-30 \mu$. Areolae arranged in straight to slightly curved, nearly parallel rows. Areolae become smaller outward, about 16 in $10 \mu$ midway. Hyaline central area with two opposite processes surrounded by slightly large and conspicuous areolae. One concentric row of strutted processes present about halfway from center to margin. One row of marginal processes surrounds the inner edge of margin, about 7 in $10 \mu$. Valve margin radially striated, about 20 striae in $10 \mu$.

Holotype: Plate 1, Figure 16, $29 \mu$ in diameter, from DSDP Leg 55, Sample 433A-6-4, 8-10 cm, North Pacific Ocean.

Paratype: Plate 1, Figure 15, $20 \mu$ in diameter, from DSDP Leg 55, Sample 433A-6-4, 8-10 cm, North Pacific Ocean.

Discussion: This species is most characterized by the presence of opposite central processes. No similar species were found in the literature.

Occurrences: Up to now found only in the upper Miocene sediment from DSDP Hole 433A.

\section{EMENDED DESCRIPTIONS OF TWO DIATOM SPECIES Genus DENTICULA Kützing (1884) \\ Denticula kamtschatica Zabelina (1934), emend. Koizumi (Plate 2, Figures 1-10)} 10.

Synonyms: Nitzschia rolandii Schrader, Harper, 1977, pl. 3, fig. 9,

Description: Simonsen and Kanaya, 1961, p. 503, pl. 1, fig. 14-18.

Emended description: The number of pseudosepta in $10 \mu$ has been expanded to 5-8. Pseudosepta irregularly branch near the raphe.

Discussion: This species is represented by specimens having 6-8 pseudosepta in $10 \mu$ in the DSDP Leg 55 samples, as well as other DSDP samples; Schrader, 1973a, pl. 2, fig. 3-13, DSDP Sites 173 and 178; Koizumi, 1973a, pl. 5, fig. 15, DSDP Site 183; Koizumi, 1975a, pl. 1, fig. 13-16, 19, 20, DSDP Sites 301 and 302. They cannot be distinguished from the type form, because of the presence of intermediate form in the number of pseudosepta in $10 \mu$.

\section{Genus NITZSCHIA Hassal (1845)}

\section{Nitzschia rolandii Schrader (1973), emend. Koizumi} (Plate 2, Figures 15-20) 42 .

Description: Schrader, 1973a, p. 708, pl. 26, fig. 3, 4, pl. 5, fig. 31,

Emended description: Valves linear to linear-lanceolate with parallel sides and bluntly rounded ends, $10-45 \mu$ in length, 3-6 $\mu$ in width. Transapical striae $10-12$ in $10 \mu$, punctation not visible in the normal light microscope. Transapical striae regularly branch near the keel, mostly share branches to one another.

Discussion: Harper (1977) referred specimens resembling Denticula kamtschatica to Nitzschia rolandii emend. Harper. However, two forms of them are considered to be Denticula kamtschatica by the expanded number of pseudosepta in $10 \mu$ and the irregular branching pattern of pseudosepta. This species, in DSDP Hole 433A samples, is more similar to the illustration of paratype than one of holotype by Schrader (1973a).

\section{FLORAL REFERENCES}

The references are given for those taxa mentioned in this report. The taxa are arranged alphabetically, separately under the marine 
planktonic and marine tychopelagic to benthic diatoms. The taxa that were treated by Hustedt $(1930,1959)$ are referred directly to his work. For the taxa that were not treated by Hustedt, references, as far as possible, are made to the original descriptions. Additional references are selected from remarks and illustrations which were helpful for the present work. Marine planktonic diatoms which are important for the biostratigraphy and taxonomy are illustrated.

\section{Marine Planktonic Diatoms}

Actinocyclus curvatulus Jan., in Schmidt, 1878: Hustedt, 1930, p. 538, fig. 307; Koizumi, 1973a, p. 831, pl. 1, fig. 1-6.

Actinocyclus divisus (Grun.) Hustedt, 1958: p. 129, pl. 8, fig. 81; Koizumi, 1973a. p. 831, pl. 1, fig. 7-12.

Actinocyclus ellipticus Grun., in Van Heurck, 1883: Hustedt, 1930, p. 533, fig. 303; Schrader, 1974, p. 908, pl. 17, fig. 4, 5, 16. [Plate 1, Figure 19.]

Actinocyclus ellipticus Grun. forma lanceolata Kolbe, 1954: p. 20, pl. 3, fig. 27; Schrader, 1974, p. 908, pl. 17, fig. 2. [Plate 1, Figure 20.]

Actinocyclus ellipticus Grun. var. elongatus (Grun.) Kolbe, 1954: p. 20, pl. 3, fig. 28, 31; Schrader, 1974, p. 908, pl. 17, fig. 3. [Plate 1, Figure 21.]

Actinocyclus ingens Rattray, 1890: p. 149, pl. 11, fig. 7; Kanaya, 1971 , p. 554, pl. 40.6, fig. 1-8; Koizumi, 1973a, p. 831, pl. 1, fig. 13, 14, pl. 2, fig. 1, 2. Remarks: Two kinds of specimens were found, those with the concentric undulation of the valve surface and those with valves almost flat. Separation into two different taxa has not been done, because they show a continuous transition from one extreme to another.

Actinocyclus ochotensis Jousé, 1968: p. 17, pl. 2, fig. 2-5; Koizumi, 1973 a, p. 831 , pl. 2, fig. $8,9$.

Actinocyclus oculatus Jousé, 1968: p. 18, pl. 2, fig. 6, 7; Koizumi, 1973a, p. 831, pl. 2, fig. 8, 9.

Actinocyclus tsugaruensis Kanaya, 1959: p. 99, pl. 8, fig. 5-8.

Asterolampra acutiloba Forti, in Tempère and Peragallo, 1912: Schrader, 1974, p. 911, pl. 9, fig. 7, 8. [Plate 1, Figure 18.].

Asterolampra grevillei (Wall.) Greville, 1860: Hustedt, 1930, p. 489, fig. 274: Schrader, 1974, p. 911, pl. 8, fig. 5.

Asterolampra marylandica Ehr., 1844: Hustedt, 1930, p. 485, fig. 270, 271: Schrader, 1974, p. 912, pl. 8, fig. 2. [Plate 2, Figure 24.]

Asteromphalus darwinii Ehr., 1844: Greville, 1860, p. 116, pl. 4, fig. 12, 13; Hanna, 1970, p. 180, fig. 90. Synonyms: Asteromphalus robustus Castr., Sheshukova-Poretzkaya, 1967, pl. 29, fig. 2; Asteromphalus $\mathrm{cf}$. robustus Castr., Schrader, 1973a, pl. 21, fig. 7. [Plate 2, Figure 26.]

Asteromphalus flabellatus (Bréb.) Grev., 1859: Hustedt, 1930, p. 498, fig. 279; Schrader, 1974, p. 912, pl. 8, fig. 3.

Asteromphalus hookeri Ehr., 1844: Hustedt, 1958, p. 127, pl. 8, fig. 88-90; Schrader, 1973a, p. 702, pl. 21, fig. 6. [Plate 2, Figure 23.]

Asteromphalus hungaricus Pantocsek, 1889: p. 111, pl. 30, fig. 436, pI. 31, fig. 451. [Plate 2, Figure 25.]

Asteromphalus robustus Castr., 1875: Hustedt, 1930, p. 496, fig. 278; Koizumi, 1975b, p. 876, pl. 3, fig. 5 .

Coscinodiscus curvatulus Grun., in Schmidt, 1878: Hustedt, 1930, p. 406, fig. 214; Fenner, Schrader, and Wienigk, 1976, p. 773, pl. 6, fig. 3-10. Remarks: Valves nearly flat, $15-30 \mu$ in diameter. Valve surface divided into sectors bounded by curved rows, the areolae running parallel to the lateral row of each sector. At the outer end of the long curved row of areolae, marginal process always situated. Areolae are irregularly distributed in the central area, slightly smaller near margin, 8-13 in $10 \mu$ midway. [Plate 2, Figures 21,22 .]

Coscinodiscus endoi Kanaya, 1959: p. 76, pl. 3, fig. 8-11; Schrader, 1973a, p. 702, pl. 7, fig. 14, 21, pl. 20, fig. 1, 2 .

Coscinodiscus lewisianus Greville, 1866: p. 78, pl. 8, fig. 8-10; Kanaya, 1971, p. 555 , pl. 40.5, fig. 4-6; Schrader, 1973a, p. 703, pl. 8, fig. 1-6, 10, 15 .

Coscinodiscus marginatus Ehr., 1843: Hustedt, 1930, p. 416, fig. 223; Koizumi, 1975b, p. 876, pl. 2, fig. 18. Remarks: Coscinodiscus marginatus Ehr. forma fossilis Jousé, 1961, p. 68, pl. 3, fig. 7, 8; Koizumi, 1973 a, p. 832 , pl. 3, fig. $12-14$, is included here.

Coscinodiscus nodulifer Schmidt, 1878: Hustedt, 1930, p. 426, fig. 229; Koizumi, 1975b, p. 876, pl. 1, fig. 3-10.
Coscinodiscus oculatus-iridis Ehr., 1839: Hustedt, 1930, p. 454, fig. 252.

Coscinodiscus radiatus Ehr., 1839: Hustedt, 1930, p. 240, fig. 225.

Coscinodiscus stellaris Rop., 1858: Hustedt, 1930, p. 395, fig. 207; Schrader, 1973a, p. 704, pl. 26, fig. 7.

Coscinodiscus symbolophorus Grunow, 1884: p. 82, pl. 4, fig. 3-6; Schrader, 1973a, p. 703, pl. 22, fig. 8, 9. Synonyms: Coscinodiscus stellaris Rop. var. symbolophorus (Grun.) Jörg., Koizumi, 1973a, p. 832 , pl. 4, fig. 5,6 .

Coscinodiscus tabularis Grun., 1884: Hustedt, 1930, p. 427, fig. 230; Koizumi, 1975b, p. 876, pl. 2, fig. 14, 15.

Cosmiodiscus insignis Jousé, 1961: p. 67, pl. 2, fig. 8; Koizumi, 1973a, p. 832, pl. 4, fig. 7-11.

Denticula hyalina Schrader, 1973: 1973a, p. 704, pl. 1, fig. 12-22; Koizumi, 1975a, p. 799, pl. 1, fig. 21-26.

Denticula lauta Bail., 1854: Simonsen and Kanaya, 1961, p. 500, pl. 1, fig. 1-8; Schrader, 1973a, p. 705, pl. 2, fig. 14-25, 35. [Plate 2, Figures 11, 12.]

Denticula punctata Schrader, 1973: 1973a, p. 705, pl. 1, fig. 25-30, pl. 3, fig. 16, 17. [Plate 2, Figures 13, 14.]

Denticula seminae Simonsen and Kanaya, 1961: p. 503, pl. 1, fig. 26-30; Koizumi, 1973a, p. 832, pl. 5, fig. 1-4.

Denticula seminae Simonsen and Kanaya var. fossilis Schrader, 1973: 1973b, p. 420, pl. 1, fig. 5, 6; Koizumi, 1975c, p. 621, pl. 1, fig. 3, 4. Synonyms: Denticula seminae Simonsen and Kanaya, Koizumi, 1973a, p. 832, pl. 5, fig. 5-13.

Hemidiscus cuneiformis Wall., 1860: Hustedt, 1930, p. 904, fig. 542; Koizumi, 1975a, p. 802, pl. 4, fig. 2.

Mediaria splendida Sheshukova-Poretzkaya, 1962: p. 210, fig. 2, 5; Koizumi, 1973a, p. 832, pl. 7, fig. 5, 6.

Nitzschia fossilis (Frenguelli) Kanaya, in Kanaya and Koizumi, 1970: p. 59; Koizumi and Kanaya, 1976, p. 155, pl. 1, fig. 11-14.

Nitzschia jouseae Burckle, 1972: p. 240, pl. 2, fig. 17-21; Schrader, 1974, p. 915, pl. 7, fig. 14-23; Koizumi, 1975b, p. 877, pl. 4, fig. 18-27. [Plate 3, Figures 9, 10.]

Nitzschia kolaczeckii Grun., 1867: Kolbe, 1955, p. 174, pl. 2, fig. 22, 23; Hasle, 1960 , p. 24, pl. 5, fig. 50b, 50c.

Nitzschia marina Grun., in Cleve and Grunow, 1880: p. 70; Kolbe, 1954, p. 40, pl. 3, fig. 38-40; Schrader, 1973a, p. 707, pl. 4, fig. 17-19.

Nitzschia miocenica Burckle, 1972: p. 240, pl. 2, fig. 10-15; Schrader, 1974, p. 915, pl. 5, fig. 10, 11. [Plate 3, Figures 15-18.]

Nitzschia oceanica Hasle, 1972: p. 115, Hasle, 1965, p. 11, pl. 1, fig. 15-19, pl. 2, fig. 6-9, pl. 3, fig. 1, 2, pl. 16, fig. 1, 2 as Fragilariopsis oceanica (C1.) Hasle. [Plate 3, Figures 11-14.]

Nitzschia reinholdii Kanaya, in Kanaya and Koizumi, 1970: p. 58; Koizumi and Kanaya, 1976, p. 155, pl. 1, fig. 15-18.

Nitzschia sicula (Cast.) Hustedt, 1958: p. 180, fig. 128-132; Hasle, 1960, p. 26, pl. 7, fig. 64, 65, 16.

Planktoniella sol (Wall.) Schütt, 1893: Hustedt, 1930, p. 465, fig. 295; Gerloff, 1970, p. 203, pl. 1-14, fig. 1-51.

Pseudoeunotia doliolus (Wall.) Grun., 1880: Hustedt, 1959, p. 258, fig. 737; Koizumi and Kanaya, 1976, p. 155, pl. 1, fig. 9, 10.

Rhizosolenia barboi Brun, 1894: Schrader, 1973a, p. 709, pl. 24, fig. 4, 7; Koizumi, 1975b, p. 877, pl. 4, fig. 52, 53. Synonyms: Rhizosolenia curvirostris Jousé, Koizumi, 1975a, pl. 1, fig. 35-37; Koizumi and Kanaya, 1976, p. 155, pl. 1, fig. 19. Rhizosolenia curvirostris Jousé var. inermis Jousé, Koizumi, 1973a, p. 833, pl. 5, fig. $32,33$.

Rhizosolenia bergonii Perag., 1892: Hustedt, 1930, p. 575, fig. 327; Koizumi and Kanaya, 1976, p. 155, pl. 1, fig. 20, 21.

Rhizosolenia curvirostris Jousé, 1968: p. 19, pl. 3, fig. 2; Koizumi, 1973a, p. 833, pl. 5, fig. 29-31.

Rhizosolenia hebetata (Bail.) Gran forma hiemalis Gran, 1904: Hustedt, 1930, p. 590, fig. 337; Koizumi, 1973a, p. 833, pl. 5, fig. 34, 35.

Rhizosolenia styliformis Brig., 1858: Hustedt, 1930, p. 584, fig. 333; oizumi, 1975a, p. 802, pl. 1, fig. 33.

Rhizosolenia sp. a Koizumi, 1968: p. 217, pl. 34, fig. 22a, 22b; Koizumi, 1975a, p. 802, pl. 1, fig. 33. Remarks: Thickened walls of spine twisted.

Rhizosolenia sp. e Koizumi, 1968: p. 217, pl. 34, fig. 26; Koizumi, 1973a, p. 833, pl. 5, fig. 39. Remarks: Apical process slightly bent, with a cavity expanded in the middle part of the process. 
Rhizosolenia sp. f Koizumi. Synonyms: Rhizosolenia hebetata (Bail.) Gran forma hiemalis Gran, Koizumi, 1975a, p. 802, pl. 1, fig. 32. Remarks: Spine of apical process entirely twisted.

Roperia tesselata (Roper.) Grun., in Van Heurck, 1880: Hustedt, 1930 , p. 523, fig. 297; Koizumi, 1975 b, p. 877 , pl. 2, fig. 3, 4.

Rouxia californica Perag., in Tempère and Peragallo, 1910: p. 117, Hanna, 1930, p. 186, pl. 14, fig. 6, 7; Koizumi, 1975a, p. 802, pl. 1 , fig. 52.

Rouxia naviculoides Schrader, 1973: 1973a, p. 710, pl. 3, fig. 27-32.

Rouxia yabei Hanna, 1930: p. 185, pl. 15, fig. 2-4; Schrader, 1973a, p. 710 , pl. 3 , fig. $21,23$.

Stephanopyxis turris (Grev. and Arn.) Ralfs, in Pritchard, 1861: Hustedt, 1930, p. 304, fig. 140; Koizumi, 1973a, p. 833, pl. 6, fig. 13-16.

Synedra jouseana Sheshukova-Poretzkaya, 1962: p. 208, fig. 4; Koizumi, 1973a, p. 833, pl. 6, fig. 17.

Thalassionema nitzschioides Grun., 1881: Hustedt, 1959, p. 244, fig. 725 ; Koizumi, 1975a, p. 803, pl. 1, fig. 50, 51.

Thalassionema nitzschioides Grun. vars. Remarks: Following three varieties are presented: Thalassionema nitzschioides Grun. var. inflata Heiden and Kolbe, 1928, p. 564, pl. 35, fig. 116; Thalassionema nitzschioides Grun. var. incurvata Heiden and Kolbe, 1928, p. 564, pl. 35, fig. 117; Thalassionema nitzschioides Grun. var. parva Heiden and Kolbe, 1928, p. 564, pl. 35, fig. 118.

Thalassiosira antiqua (Grun.) Cleve-Euler, 1941: p. 173, fig. 4, 5, 74-84; Koizumi, 1975a, p. 803, pl. 4, fig. 9.

Thalassiosira aff. borealis Koizumi. Remarks: Valves almost flat, with rounded margins. Diameter of valves 18-33 $\mu$. Areolae form irregular radial rows, secondary more or less concave outwardly, giving irregularly sectorial pattern. Areolae about 8 in $10 \mu$, nearly same size on entire valve. One row of inconspicuous marginal processes near the valve margin. A more thorough study is necessary to establish the relationship among Thalassiosira borealis Koizumi (this paper), Thalassiosira burcklina Schrader, 1974, p. 916, pl. 1, fig. 21-26, and Thalassiosira aff. borealis Koizumi. [Plate 1, Figures 15-17.]

Thalassiosira convexa Mukhina, 1965: p. 22, pl. 11, fig. 1, 2; Koizumi, 1975a, p. 803, pl. 4, fig. 15-20. Remarks: Thalassiosira convexa Mukhina var. aspinosa Schrader, 1974, p. 916, pl. 2, fig. $8,9,13 \mathrm{a}-21$, is included here.

Thalassiosira decipiens (Grun.) Jörg., 1905: Hustedt, 1930, p. 322, fig. 158; Koizumi, 1973a, p. 834, pl. 7, fig. 16-18.

Thalassiosira eccentrica (Ehr.) Cleve, 1904: Sheshukova-Poretzkaya, 1967, p. 141, pl. 14, fig. 4; Fryxell and Hasle, 1972, p. 297, pl. 1-4, fig. 1a-18. Synonyms: Coscinodiscus excentricus Ehr., Koizumi, 1973a, pl. 2, fig. 11, 12.

Thalassiosira eccentrica (Ehr.) Cl. var. fasciculata (Hust.). Synonyms: Coscinodiscus excentricus Ehr, var. fasciculata Hust., Koizumi, 1975a, pl. 2, fig. 20. Remarks: The pattern of radial bundles is more conspicuous than others as a primary sculpture.

Thalassiosira eccentrica (Ehr.) Cl. var. jousei (Kanaya). Synonyms: Coscinodiscus excentricus Ehr. var. jousei Kanaya, Koizumi, 1973a, pl. 3, fig. 1-6.

Thalassiosira eccentrica (Ehr.) C1. var. leasareolatus (Kanaya). Synonyms: Coscinodiscus excentricus Ehr. var. leasareolatus Kanaya, Koizumi, 1973a, pl. 3, fig. 7, 11; Koizumi, 1975a, pl. 3, fig. 5. Remarks: The meshwork of areolae is loose in the center of the valves and a few smaller areolae are present in the hyaline center.

Thalassiosira gravida Cl., 1896: Hustedt, 1930, p. 325, fig. 161; Koizumi, 1973a, p. 834, pl. 7, fig. 19-21.

Thalassiosira gravida Cl. var. fossilis Jousé, 1961: p. 63, pl. 1, fig. 9; Koizumi, 1973a, p. 834, pl. 7, fig. 22-24.

Thalassiosira hyalina (Grun.) Gran, 1897: Hustedt, 1930, p. 323, fig. 159; Koizumi, 1973a, p. 834, pl. 8, fig. 1, 2.

Thalassiosira leptopus (Grun.) Hasle and Fryxell, 1977: p. 20, fig. 114. Synonyms: Coscinodiscus lineatus Ehr., Koizumi, 1975b, pl. 2, fig. 5,6 .

Thalassiosira lineata Jousé, 1968: p. 13, pl. 1, fig. 1, 2: Koizumi, 1975 b, p. 877, pl. 2, fig. 9-12.

Thalassiosira miocenica Schrader, 1974: p. 916, pl. 22, fig. 1-5, 11113.

Thalassiosira oestrupii (Ostf.) Proshkina-Lavrenko, 1956: Hasle, 1960 , p. 8, pl. 1, fig. 5-7; Koizumi, 1975a, p. 803, pl. 5, fig. 3, 4.

Thalassiosira plicata Schrader, 1974: p. 917, pl. 3, fig. 1, 2, 4-, 7-9.
Synonyms: Actinocyclus cf. curvatulus Jan., Schrader, 1974, pl. 3, fig. 3. Remarks: In the DSDP Leg 55 materials, aerolae are smaller, 14-17 in $10 \mu$ midway and a few subangular areolae present in the plicated central area.

Thalassiosira praeconvexa Burckle, 1972: p. 242, pl. 2, fig. 7-9; Koizumi, 1975 b, p. 878, pl. 3, fig. 21-28.

Thalassiothrix longissima Cl. and Grun., 1880: Hustedt, 1930, p. 247, fig. 726; Hasle and de Mendiola, 1967, p. 114, fig. 20.

\section{Marine Tychopelagic-Benthic Diatoms}

Actinocyclus ehrenbergii Ralfs, in Pritchard, 1861: Hustedt, 1930, p. 525, fig. 298-302; Koizumi, 1973b, p. 134, pl. 20, fig. 10, 11.

Actinocyclus ehrenbergii Ralfs var. tenella (Bréb.) Hustedt, 1930: p. 530, fig. 302; Schrader, 1974, p. 908, pl. 20, fig. 1, 3.

Actinoptychus undulatus (Bail.) Ralfs, in Pritchard, 1861: Hustedt, 1930 , p. 475 , fig. 264; Koizumi, 1973b, p. 134, pl. 20, fig. 1a-3b.

Cocconeis costata Greg., 1855: Hustedt, 1959, p. 332, fig. 785; Sheshukova-Poretzkaya, 1967, p. 262, pl. 44, fig. 4a-4B.

Diploneis bombus Ehr., 1844: Hustedt, 1959, p. 704, fig. 1086.

Rhabdonema japonicum Tem. and Brun, in Brun and Tempère, 1889: p. 53, pl. 1, fig. 6; Sheshukova-Poretzkaya, 1967, p. 258, pl. 43, fig. 14, pl. 44, fig. 1a-1; Hanna, 1970, p. 192, fig. 47, 92, 93, 95.

\section{REFERENCES}

Bailey, J. W., 1854. Notes on new species and localities of microscopical organisms, Smithsonian Inst., Contrib. Knowl., v. 7 , pp. 1-15.

Brightwell, T., 1858. Remarks on the genus Rhizosolenia of Ehrenberg, Quart. J. Micro. Sci., v. 6, pp. 1-93.

Brun, J., 1894. Espèces nouvelles, Le diatomiste, v. 2, pp. 7278.

Brun, J. and Tempère, J., 1889. Diatomées fossiles du Japon. Espéces marines et nouvelles des calcaires argileux de Sendai and de Yedo, Mèm. Soc. Phys. d'Hist. Nat. Genève, v. 30, pp. 1-75.

Burckle, L. H., 1972. Late Cenozoic planktonic diatom zones from the eastern equatorial Pacific, Nova Hedwigia, v. 39, pp. 217-250.

manuscript. Early Miocene to Pliocene diatom datum levels for the equatorial Pacific.

Burckle, L. H. and Opdyke, N. D., 1977. Late Neogene diatom correlations in the circum-Pacific. In Saito, T. and Ujiie, H. (Eds.), Proceedings of the First International Congress on Pacific Neogene Stratigraphy, Tokyo, 1976: Tokyo (Kaiyo Shuppan Co. Ltd.), pp. 255-284.

Castracane, Conte A. F. A., 1875. Contribuzione alla florula delle Diatomee del Mediterraneo ossia esame del contenuto dello stomaco di una Salpa pinnata, pescata a Messina, Atti. Acad. Pontif dei nouvi Lincei., v. 28, pp. 377-396.

Cleve, P. T., 1873. Examination of diatoms found on the surface of the Sea of Japan, Kgl. Svensk. Vetensk. Akad. Handl., v. 11, pp. 1-13.

1896. Diatoms from Buffin Bay and Davis Strait, Bihang Kgl. Svensk. Vetensk. Akad. Handl., Abt. 3, v. 22, pp. $1-22$.

1904. Plankton table for the North Sea, Bull. Cons. Explor. Mer. 1903-1904, pp. 1-216.

Cleve, P. T. and Grunow, A., 1880. Beitrage zur Kenntnis der Arktischen Diatomeen, Kgl. Svensk. Vetensk. Akad. Handl., v. 17, pp. 13-122.

Cleve-Euler, A., 1941. Alltertiäre Diatomeen und Silicoflagellaten im Junneren Schwedes, Paleontography, v. 92, pp. 166-209.

Ehrenberg, C. G., 1839. Über noch jetzt zahlreich lebende Tierarten der Kreidebildung und den Organismus der Polythalamien, Abh. Kgl. Akad. Wiss. Berlin, pp. 81-174. 1843. Mitteilung über 2 neue asiatische Lager fossifossiler Infusorien-Erden aus dem russischen Trans- 
Kaukasien (Grusien) und Sibirien, Ber über die zur Bekanntmachung geeigneten Verhdlg. der Königl. preuss. Acad. d. Wissensch. zu Berlin, pp. 43-49.

1844. Resultate Untersuchungen der ihm von der Sudpolreise des Kapt. Rofs in den Jahren 1841-1843, Ber. Berl. Akad. Wiss., pp. 182-207.

Fenner, J., Schrader, H. J., and Wienigk, H., 1976. Diatom phytoplankton studies in the Southern Pacific Ocean; composition and correlation to the Antarctic Convergence and its paleoecological significance. In Hollister, C. D., Craddock, C., et al., Initial Reports of the Deep Sea Drilling Project, v. 35: Washington (U. S. Gov't. Printing Office), pp. 757-814.

Fryxell, G. A. and Hasle, G. R., 1972. Thalassiosira eccentrica (Ehrenb.) Cleve, T. symmetrica sp. nov., and some related centric diatoms, J. Phycology, v. 8, pp. 297-317.

Gerloff, J., 1970. Elektronenmikroskopische Untersuchungen an Diatomeenschalen 7; Der Bau der Schale von Planktoniella sol (Wallich) Schütt, Nova Hedw. Beihft., v. 31, pp. 203-234.

Gran, H. H., 1897. Protophyta: Diatomaceae, Silicoflagellata and Cilioflagellata, Den. Norske Nordhavs-Exped., 18761878, Bot., Hefte 24, pp. 1-36.

Gregory, W., 1855. On a post-Tertiary lacustrine sand, containing diatomaceous exuviae, from Glenshira, near Inverary, Quart. J. Micr. Sci., v. 3, pp. 30-43.

Greville, R. K., 1859. Descriptions of diatomaceae observed in Californian Guano, Quart. J. Micr. Sci., v. 7, pp. 155-166. 1860. A monograph of the genus Asterolampra including Asteromphalus and Spatangidium, Trans. Microscop. Soc. London, n. ser., v. 8, pp. 102-124. 1866. Descriptions of new and rare diatoms, Trans. Microscop. Soc. London, n. ser., v. 19, pp. 77-88.

Grunow, A., 1867. Nachträgliche Bemerkungen über die von Herrn Ländig gesammelton Diatomeen von Honduras, Hedwigia, v. 6, pp. 33-37. 1880. On some new species of Nitzschia, J. R. Micr. Soc., v. 3, pp. 394-397.

1884. Die Diatomeen von Franz Josefs-Land, Denkschr. Kgl. Akad. Wiss. Wien, Math. Nat. Wiss. K1., v. 48, pp. $53-112$.

Hanna, G. D, 1930. Review of the genus Rouxia, J. Paleontol., v. 4, pp. 179-188.

1970. Fossil diatoms from the Pribilof Islands, Bering Sea, Alaska, Proc. Calif. Acad. Sci., 4th ser., v. 37, pp. 167-234.

Harper, H. E., Jr., 1977. Diatom biostratigraphy. In Ujiie, H., Saito, T., Kent, D. V., Thompson, P. R., Okada, H., Klein, G. dV., Koizumi, I., Harper, H. E., Jr., and Sato, T. (Eds.), Biostratigraphy, Paleomagnetism and Sedimentology of Late Cenozoic Sediments in Northwestern Hokkaido, Japan: Bull. National Sci. Museum, ser. C (Geol.), v. 3, pp. 85-90.

Hasle, G. R., 1960. Phytoplankton and cilate species from the tropical Pacific, Skrift. det Norske Videnskaps-Akad. Oslo, Matemat.-Naturv., v. 2, pp. 1-50.

1965. Nitzschia and Fragilariopsis species studied in the light and electron microscopes; III. The genus Fragilariopsis. Skr. Norske. Vidensk. Akad. I. Mat.-Nat. K1. $n$. ser., v. 21, pp. 1-46.

1972. Fragilariopsis Hustedt as a section of the genus Nitzschia Hassall, Nova Hedwigia, v. 39, pp. 111-120. 1973. Thalassiosiraceae, a new diatom Family, Norw. J. Bot., v. 20, pp. 67-69.

Hasle, G. R. and de Mendiola, B. R. E., 1967. The fine structure of some Thalassionema and Thalassiothrix species, Phycologia, v. 6, pp. 107-125.
Hasle, G. R. and Fryxell, G. A., 1977. The genus Thalassiosira; some species with a linear areola array, Nova Hedwigia, v. 54, pp. 15-66.

Hasle, G. R. and Heimdal, B. R., 1968. Morphology and distribution of the marine centric diatom Thalassiosira antarctica Comber, J. Roy. Microscop. Soc., v. 88, pp. 357-369.

Hassal, A. H., 1845. A History of the British Freshwater Algae (Including Descriptions of Diatomaceae and Desmidiaceae) with Upwards of One Hundred Plates: London (Taylor, Walton and Maberly), v. 1, 462 pp.

Heiden, H. and Kolbe, R. W., 1928. Die Marinen Diatomeen der Deutschen Südpolar-Expedition, 1901-1903. In Deutschen Südpolar-Expedition, 1901-1903. Berlin (Botanik), v. 8, pp. 449-745.

Hendey, N. I., 1937. The plankton diatoms of the southern seas, Discovery Rept., v. 16, pp. 151-364.

Hustedt, F., 1930. Die Kieselalgen Deutschland, Osterreichs und der Schweiz. In Rabenhorst, L. (Ed.), KryptogamenFlora von Deutschland, Osterreichs und der Schweiz. Leipzig, v. 7, pt. 1,920 pp.

1958. Diatomeen aus der Antarktis und dem Südatlantik. In Deutsche Antarktische Exped. 1938-1939, Wiss. Exped: Hamburg, v. 2, pp. 103-191.

1959. Die Kieselalgen Deutschland, Osterreichs und der Schweiz. In Rabenhorst, L. (Ed.), Kryptogamen-Flora von Deutschland, Osterreichs und der Schweiz. Leipzig, v. 7, pt. 2, 845 pp.

Jörgensen, E., 1905. The protist plankton and the diatoms in bottom samples, Bergens Mus. Skr., v. 7, pp. 49-148.

Jousé, A. P., 1961. Miocene and Pliocene marine diatoms from the Far East, Bot. Mater. Spor. Rast., Bot. Inst., Akad. Nauk SSSR, v. 16, pp. 59-70.

1968. New species of diatoms in bottom sediments of the Pacific and the Sea of Okhotsk, Nov. Systemat. Plant. non Vascular 1968, Akad. Nauk SSSR, v. 3, pp. 12-21.

Kanaya, T., 1959. Miocene diatom assemblages from the Onnagawa Formation and their distribution in the correlative formations in northeast Japan, Sci. Rep. Tohoku Univ., 2nd ser (Geol.), v. 30, pp. 1-30.

1971. Some aspects of pre-Quaternary diatoms in the oceans. In Funnell, B. M. and Riedel, W. R. (Eds.), The Micropaleontology of Oceans: Cambridge (Cambridge Univ. Press), pp. 545-565.

Kanaya, T. and Koizumi, I., 1966. Interpretation of diatom thanatocoenoses from the North Pacific applied to a study of core V20-130 (Studies of a deep-sea core V20-130, part IV), Sci. Rep. Tohoku Univ., 2nd ser. (Geol.), v. 37, pp. 89-130.

1970. The progress in the younger Cenozoic diatom stratigraphy in the northern circum-Pacific region, $J$. Marine Geol., v. 6, pp. 47-66.

Koizumi, I., 1968. Tertiary diatom flora of Oga Peninsula, Akita Prefecture, northeast Japan, Sci. Rep. Tohoku Univ., 2nd ser. (Geol.), v. 40, pp. 171-225.

1973a. The Late Cenozoic diatoms of Sites 183-193, Leg 19, Deep Sea Drilling Project, In Creager, J. S., Scholl, D. W., et al., Initial Reports of the Deep Sea Drilling Project, v. 19: Washington (U. S. Gov't. Printing Office), pp. 805-855.

1973b. Marine diatom flora of the Pliocene Tatsunokuchi Formation in Miyagi Prefecture, Palaeont. Soc. Japan Trans. Proc., v. 79, pp. 126-136.

1975a. Neogene diatoms from the western margin of the Pacific Ocean, Leg 31, Deep Sea Drilling Project. In Karig, D. E., Ingle, J. C., Jr., et al. Initial Reports of the 
Deep Sea Drilling Project, v. 31: Washington (U. S. Gov't. Printing Office), pp. 779-819.

1975b. Neogene diatoms from the northwestern $\mathrm{Pa}-$

cific Ocean, Deep Sea Drilling Project. In Larson, R. L.,

Moberly, R., et al., Initial Reports of the Deep Sea Drilling Project, v. 32: Washington (U. S. Gov't. Printing Office), pp. 865-889.

1975c. Late Cenozoic diatom biostratigraphy in the circum-North Pacific region, J. Geol. Soc. Japan, v. 81, pp. 611-627.

1977. Diatom biostratigraphy in the North Pacific region. In Saito, T. and Ujiie, H. (Eds.), Proceedings of the First International Congress on Pacific Neogene Stratigraphy, Tokyo, 1976: Tokyo (Kaiyo Shuppan Co. Ltd.), pp. 235-253.

Koizumi, I. and Kanaya, T., 1976. Late Cenozoic marine diatom sequence from the Choshi district, Pacific coast, central Japan. In Takayanagi, Y. and Saito, T. (Eds.), Progress in Micropaleontology: New York (Micropaleontol. Press), pp. 144-159.

Kolbe, R. W., 1954. Diatoms from equatorial Pacific cores Rept. Swedish Deep Sea Exped., Goteborg, v. 6, pt. 2, 49 pp. 1955. Diatoms from equatorial Atlantic cores Rept. Swedish Deep Sea Exped., Goteborg, v. 7, pt. 3, pp. 151-184.

Kützing, F. T., 1844. Die Kieselschaligen Bacillarien oder Diatomeen. Nordhausen, $152 \mathrm{pp}$.

Mukhina, V. V., 1965. New species of diatoms from the bottom sediments of the equatorial region of the Pacific, Nov. Systemat. Plant. non Vascular 1965, Akad. Nauk SSSR, v. 11, pp. 22-25.

Pantocsek. J., 1889. Beiträge zur Kenntnis der Fossilen Bacillarien Ungarns; Brackwasser Bacillarien, Anhang: Analyse der Marinen Depots von Bory, Bremia, Nagy-Kürtos in Ungarn; Ananino und Kusnetz in Russland, Pt. 2, 123 pp.

Peragallo, H., 1892. Monographie du genere Rhizosolenia, Le Diatomiste, v. 1, pp. 99-117.

Pritchard, A., 1861. A History of Infusoria, including the Desmidaceae and Diatomaceae, British and Foreign. 4th Ed., London (Whittaker \& Co.), 40 pp.

Proschkina-Lavrenko, A. I., 1956. De specie nova Thalassiosira e Mari Maeotico, Not. Inst. Cryptog. Bot. Acad. Sci. URSS, v. 11, pp. 57-60.

Rattray, J., 1890. A revision of the genus Actinocyclus Ehr., J. Quekett Micros. Club, v. 4, ser. 2, pp. 137-212.
Roper, F. C. S., 1858. Notes on some new species and varieties of British marine diatomaceae, Quart. J. Micr. Sci., v. 6, pp. 17-25.

Schmidt, A., 1878. Atlas der Diatomaceenkunde, Zweite revidirte Auflage. Leipzig (R. Reisland), Ser. 2, Heft 15-16, pl. 57-64.

Schrader, H. J., 1973a. Cenozoic diatoms from the northeast Pacific, Leg 18. In Kulm, L. D., von Huene, R., et al., Initial Reports of the Deep Sea Drilling Project, v. 18: Washington (U. S. Gov't. Printing Office), pp. 673-797. 1973b. Stratigraphic distribution of marine Denticula species in Neogene North Pacific sediments, Micropaleontol., v. 19, pp. 417-430.

1974. Cenozoic marine planktonic diatom stratigraphy of tropical Indian Ocean. In Fisher, R. L., Bunce, E. T., et al., Initial Reports of the Deep Sea Drilling Project, v. 24: Washington (U. S. Gov't. Printing Office), pp. 887-967.

Schrader, H. J. and Fenner, J., 1976. Norwegian Sea Cenozoic diatom biostratigraphy and taxonomy. In Talwani, M., Udintsev, G., et al., Initial Reports of the Deep Sea Drilling Project, v. 38: Washington (U. S. Gov't. Printing Office), pp. 921-1099.

Schütt, F., 1893. Das Pflanzenleben der Hochsee. Kiel und Leipzig, $76 \mathrm{pp}$.

Sheshukova-Poretzkaya, V. S., 1962. New and rare diatoms from formations of Sakhalin, Leningrad Gos. Univ., Vest. no. 313, Biol. Inst. ser. Biol., Nauk Vup., v. 49, pp. 1-203. , 1967. Neogene Marine Diatoms of Sakhalin and Kamtschatica: Leningrad (Leningrad Univ.), 327 pp.

Simonsen, R. and Kanaya, T., 1961. Note on the marine species of the diatom genus Denticula Kutz., Int. Revue. Ges. Hydrobiol., v. 46, pp. 498-513.

Tempère, J. and Peragallo, H., 1912. Diatomées du Monde Entier. 2nd Ed. 30 fasc., Arcachon, Grez-sur-Loing (S.-et-M), pt. 1 , pp. 305-352

Van Heurck, H., 1880. Synopsis des Diatomées de Belgique. Atlas, Anvers (Ducaju \& Cie), pl. 1-30.

1883. Synopsis des Diatomées de Belgique. Atlas, Anvers (Ducaju \& Cie), pl. 104-132.

Wallich, G. C., 1860. On the siliceous organisms found in the digestive cavities of the Salpae and their relations to the flint nodules of the Chalk Formation, Trans. Microsc. Soc. London, v. 8, pp. 36-55.

Zabelina, M. M., 1934. Diatoms from the Tertiary deposits of the eastern coast of Kamtschatica, Transact. Oil Geol. Inst., ser. $A, E d$. 48, pp. 3-19. 

PLATE 1

(Magnification $1500 \times$ )

Figures 1-6 Nitzschia suikoensis Koizumi, n. sp.

1, 2. Slide no. $3679,30 \mu$ in length, from DSDP Sample 433A-6-6, 8-10 cm, Denticula kamtschatica Zone, upper Miocene (Paratype).

3, 4. Slide no. $3659,41 \mu$ in length, from DSDP Sample 433A-5-5, 8-10 cm, Denticula kamtschatica Zone, upper Miocene (Holotype).

5, 6. Slide no. 3659, $60 \mu$ in length, from DSDP Sample 433A-5-5, 8-10 cm, Denticula kamtschatica Zone, upper Miocene.

Figures 7-10 Thalassiosira borealis Koizumi, n. sp.

7. Slide no. 3669, $17 \mu$ in diameter, from DSDP Sample 433A-6-3, 8-10 cm, Denticula kamtschatica Zone, upper Miocene (Holotype).

8. Slide no. 3659. $21 \mu$ in diameter, from DSDP Sample 433A-5-5, 8-10 cm, Denticula kamtschatica Zone, upper Miocene (Paratype).

9, 10. Slide no. 3671, $18 \mu$ in diameter, from DSDP Sample 433A-6-4, 8-10 cm, Denticula kamtschatica Zone, upper Miocene.

Figures 11-14 Thalassiosira jacksonii Koizumi and Barron, n. sp.

11. Slide no. 3669, $15 \mu$ in diameter, from DSDP Sample 433A-6-3, 8-10 cm, Denticula kamtschatica Zone, upper Miocene.

12. Slide no. $3643,16 \mu$ in diameter, from DSDP Sample 433A-4-5, 8-10 cm, Denticula kamtschatica Zone, upper Miocene (Holotype).

13. Slide no. $3643,20 \mu$ in diameter, from DSDP Sample 433A-4-5, 8-10 cm, Denticula kamtschatica Zone, upper Miocene.

14. Slide no. 3695, $20 \mu$ in diameter, from DSDP Sample 433-1, CC, Denticula kamtschatica-Denticula seminae var. fossilis Zone, Pliocene (Paratype).

Figures 15-17 Thalassiosira opposita Koizumi, n. sp.

15. Slide no. 3645, $20 \mu$ in diameter, from DSDP Sample 433A-4-6, 8-10 cm, Denticula kamtschatica Zone, upper Miocene (Paratype).

16. Slide no. $3671,29 \mu$ in diameter, from DSDP Sample 433A-6-4, 8-10 cm, Denticula kamtschatica Zone, upper Miocene (Holotype).

17. Slide no. $3643,17 \mu$ in diameter, from DSDP Sample 433A-4-5, 8-10 cm, Denticula kamtschatica Zone, upper Miocene.

Figure 18 Asterolampra acutiloba Forti.; Slide no. 3667, $40 \mu$ in diameter from DSDP Sample 433A-6-2, 8-10 cm, Denticula kamtschatica Zone, upper Miocene.

Figure 19 Actinocyclus ellipticus Grun.; Slide no. 3649, $35 \mu$ in length, from DSDP Sample 433A-5-1, 8-10 cm, Denticula kamtschatica Zone, upper Miocene.

Figure 20 Actinocyclus ellipticus Grun. f. lanceolata Kolbe; Slide no. 3645, $52 \mu$ in length, from DSDP Sample 433A-4-6, 8-10 cm, Denticula kamtschatica Zone, upper Miocene.

Figure 21 Actinocyclus ellipticus Grun. v. elongatus (Grun.) Kolbe; Slide no. 3645, $86 \mu$ in length, from DSDP Sample 433A-4-6, 8-10 cm, Denticula kamtschatica Zone, upper Miocene. 
PLATE 1
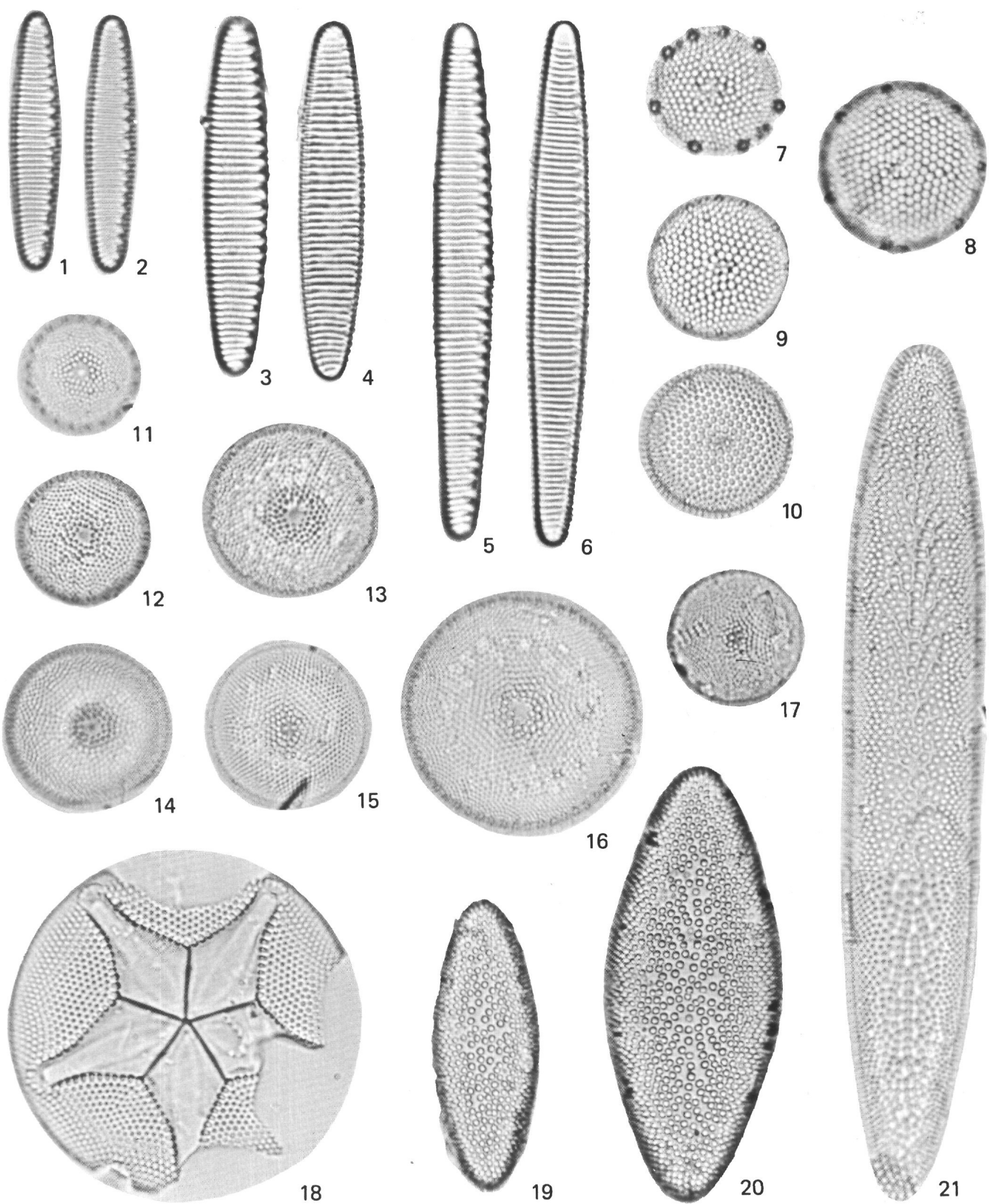
PLATE 2

(Magnification $1500 \times$ )

Figures 1-10 Denticula kamtschatica Zabelina, (emend.) Koizumi

1. Slide no. 3659, $15 \mu$ in length, from DSDP Sample 433A-5-5, 8-10 cm, Denticula kamtschatica Zone, upper Miocene (pseudosepta 5 in $10 \mu$ ).

2. Slide no. 3659, $19 \mu$ in length, from DSDP Sample 433A-5-5, 8-10 cm, Denticula kamtschatica Zone, upper Miocene (pseudosepta 4 in $10 \mu$ ).

3. Slide no. $3659,15 \mu$ in length, from DSDP Sample 433A-5-5, 8-10 cm, Denticula kamtschatica Zone, upper Miocene (pseudosepta 7 in $10 \mu$ ).

4. Slide no. 3615, $17 \mu$ in length, from DSDP Sample 433A-2-2, 28-30 cm, Denticula kamtschatica-Denticula seminae v. fossilis Zone, lower Pliocene (pseudosepta 6-7 in $10 \mu$ ).

5. Slide no. $3659,19 \mu$ in length, from DSDP Sample 433A-5-5, 8-10 cm, Denticula kamtschatica Zone, upper Miocene (pseudosepta 7 in $10 \mu$ ).

6. Slide no. $3647,30 \mu$ in length, from DSDP Sample 433A-4,CC, Denticula kamtschatica Zone, upper Miocene (pseudosepta 6-7 in $10 \mu$ ).

7. Slide no. $3619,12 \mu$ in length, from DSDP Sample 433A-3-1, 8-10 cm, Denticula kamtschatica Zone, lower Pliocene (pseudosepta 7 in $10 \mu$ ).

8. Slide no. $3615,12 \mu$ in length, from DSDP Sample 433A-2-2, 28-30 cm, Denticula kamtschatica-Denticula seminae v. fossilis Zone, lower Pliocene (pseudosepta 7-8 in $10 \mu$ ).

9. Slide no. 3613, $28 \mu$ in length, from DSDP Sample 433A-2-1, 27-29 cm, Denticula kamtschatica-Denticula seminae v. fossilis Zone, lower Pliocene (pseudosepta 8 in $10 \mu$ ).

10. Slide no. 3615, $25 \mu$ in length, from DSDP Sample 433A-2-2, 28-30 cm, Denticula kamtschatica-Denticula seminae v. fossilis Zone, lower Pliocene (pseudosepta 7 in $10 \mu)$.

Figures 11, 12 Denticula lauta Bail.; Slide no. 3734, $23 \mu$ in length, from DSDP Sample 433A-6-7, 6-7 $\mathrm{cm}$, Denticula lauta Zone, middle Miocene.

Figures 13, 14 Denticula punctata Schrader; Slide no. 3734, $22 \mu$ in length, from DSDP Sample 433A-6-7, 6-7 cm, Denticula lauta Zone, middle Miocene.

Figures 15-20 Nitzschia rolandii Schrader, (emend.) Koizumi

15,16 . Slide no. $3651,39 \mu$ in length, from DSDP Sample 433A-5-2, 8-10 cm, Denticula kamtschatica Zone, upper Miocene.

17, 18. Slide no. $3659,15 \mu$ in length, from DSDP Sample 433A-5-5, 8-10 cm, Denticula kamtschatica Zone, upper Miocene.

19, 20. Slide no. $3665,20 \mu$ in length, from DSDP Sample 433A-6-1, 8-10 cm, Denticula kamtschatica Zone, upper Miocene.

Figures 21, 22 Coscinodiscus curvatulus Grun.

21. Slide no. $3671,17 \mu$ in diameter, from DSDP Sample 443A-5-2, 8-10 cm, Denticula kamtschatica Zone, upper Miocene.

22. Slide no. $3619,23 \mu$ in diameter, from DSDP Sample 433A-3-1, 8-10 cm, Denticula kamtschatica Zone, lower Miocene.

Figure 23 Asteromphalus hookeri Ehr.; Slide no. 3617, $34 \mu$ in diameter, from DSDP Sample 433A-6-1, 8-10 cm, Denticula kamtschatica Zone, lower Pliocene.

Figure 24 Asterolampra marylandica Ehr.; Slide no. 3665, $55 \mu$ in diameter, from DSDP Sample 433A-5-5, 8-10 cm, Denticula kamtschatica Zone, upper Miocene.

Figure 25 Asteromphalus hungaricus Pant.; Slide no. 3659, broken specimen, from DSDP Sample 433A-5-5, 8-10 cm, Denticula kamtschatica Zone, upper Miocene.

Figure 26 Asteromphalus darwinii Ehr.; Slide no. 3619, $53 \times 66 \mu$ in size, from DSDP Sample 433A-3-1, 8-10 cm, Denticula kamtschatica Zone, lower Pliocene. 
PLATE 2

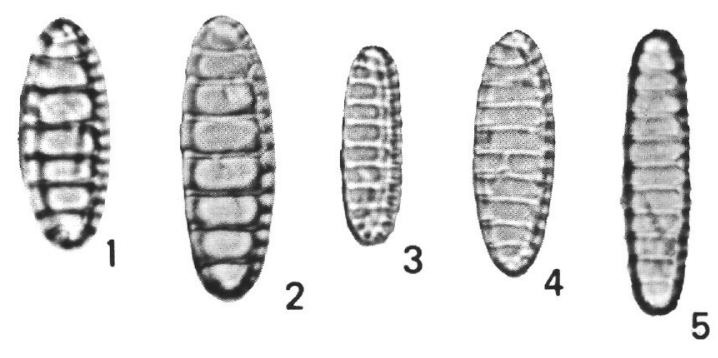

15
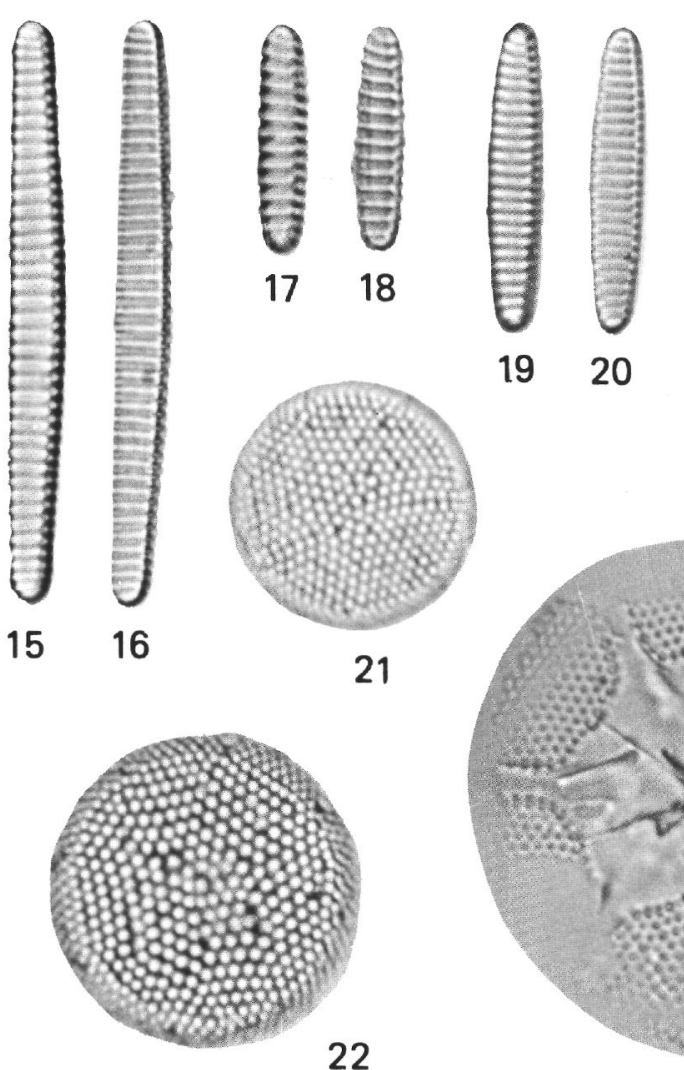

22

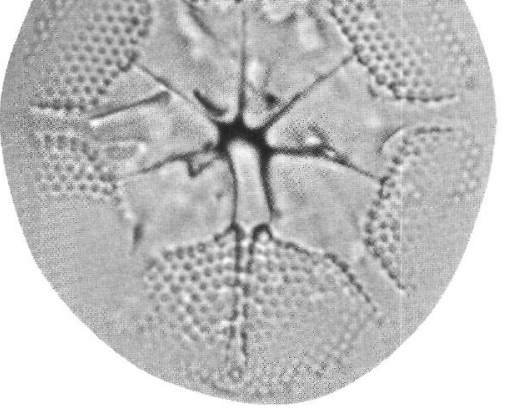

23

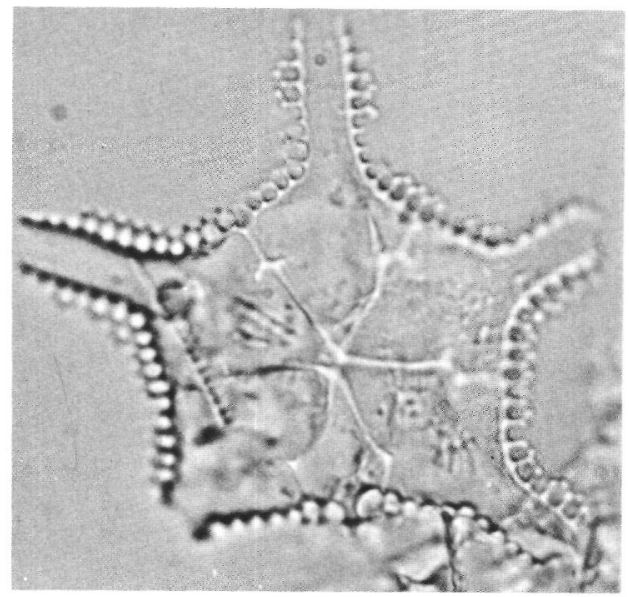

8
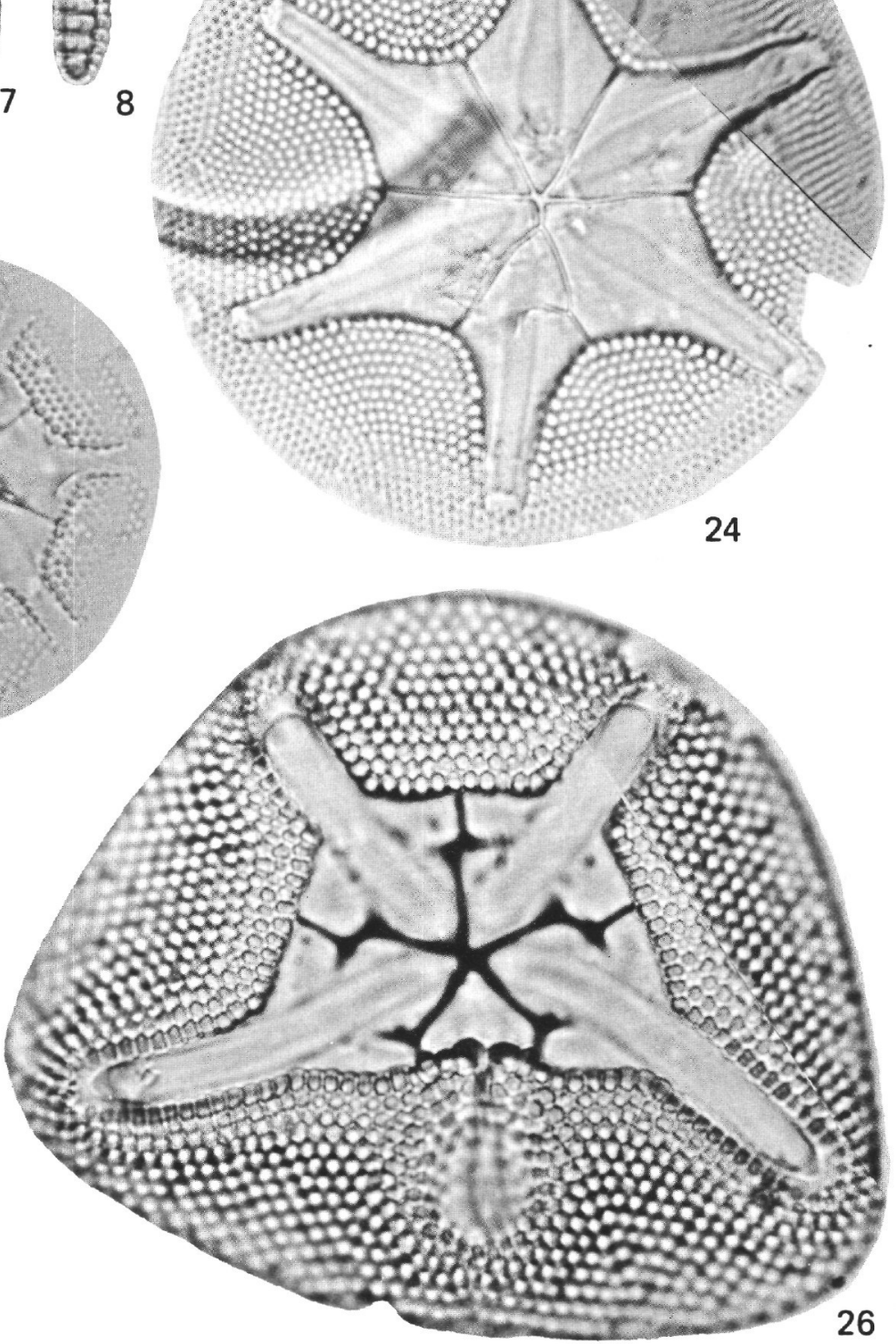
PLATE 3

(Magnification $1500 \times$ )

Figures 1-8 Nitzschia miocenica Burckle

1, 2. Slide no. $3665,17 \mu$ in length, from DSDP Sample 433A-6-1, 8-10 cm, Denticula kamtschatica Zone, upper Miocene.

3, 4. Slide no. 3679, $17 \mu$ in length, from DSDP Sample 433A-6-6, 8-10 cm, Denticula kamtschatica Zone, upper Miocene.

5, 6. Slide no. $3649,35 \mu$ in length, from DSDP Sample 433A-5-1, 8-10 cm, Denticula kamtschatica Zone, upper Miocene.

7, 8. Slide no. $3651,32 \mu$ in length, from DSDP Sample 433A-5-2, 8-10 cm, Denticula kamtschatica Zone, upper Miocene.

Figures 9-10 Nitzschia jouseae Burckle; Slide no. 3617, $29 \mu$ in length, from DSDP Sample 433A-2, CC, Denticula kamtschatica Zone, lower Pliocene.

Figures 11-14 Nitzschia cf. oceanica Hasle

11, 12. Slide no. $3645,31 \mu$ in length, from DSDP Sample 433A-4-6, 8-10 cm, Denticula kamtschatica Zone, upper Miocene.

13, 14. Slide no. 3653, $22 \mu$ in length, from DSDP Sample 433A-5-3, 8-10 cm, Denticula kamtschatica Zone, upper Miocene.

Figures 15-18 Thalassiosira miocenica Schrader

15 , 16. Slide no. $3679,18 \mu$ in diameter, from DSDP Sample 433A-6-6, 8-10 cm, Denticula kamtschatica Zone, upper Miocene.

17, 18. Slide no. 3665, $20 \mu$ in diameter, from DSDP Sample 433A-6-1, 8-10 cm, Denticula kamtschatica Zone, upper Miocene.

Figures 19-21 Thalassiosira praeconvexa Schrader

19, 20. Slide no. 3730, $17 \mu$ in diameter, from DSDP Sample 433A-6-6, 53-54 cm, Denticula kamtschatica Zone, upper Miocene.

21. Slide no. 3730, $13 \mu$ in diameter, from DSDP Sample 433A-6-6, 53-54 cm, Denticula kamtschatica Zone, upper Miocene.

Figures 22-25 Thalassiosira plicata Schrader

22, 23. Slide no. $3625,29 \mu$ in diameter, from DSDP Sample 433A-3-4, 18-20 cm, Denticula kamtschatica Zone, lower Pliocene.

24, 25. Slide no. $3621,36 \mu$ in diameter, from DSDP Sample 433A-3-2, 8-10 cm, Denticula kamtschatica Zone, lower Pliocene.

Figures 26-29 Thalassiosira aff. borealis Koizumi

26, 27. Slide no. $3665,18 \mu$ in diameter, from DSDP Sample 433A-6-1, 8-10 cm, Denticula kamtschatica Zone, upper Miocene.

28, 29. Slide no. $3643,26 \mu$ in diameter, from DSDP Sample 433A-4-5, 8-10 cm, Denticula kamtschatica Zone, upper Miocene. 
PLATE 3
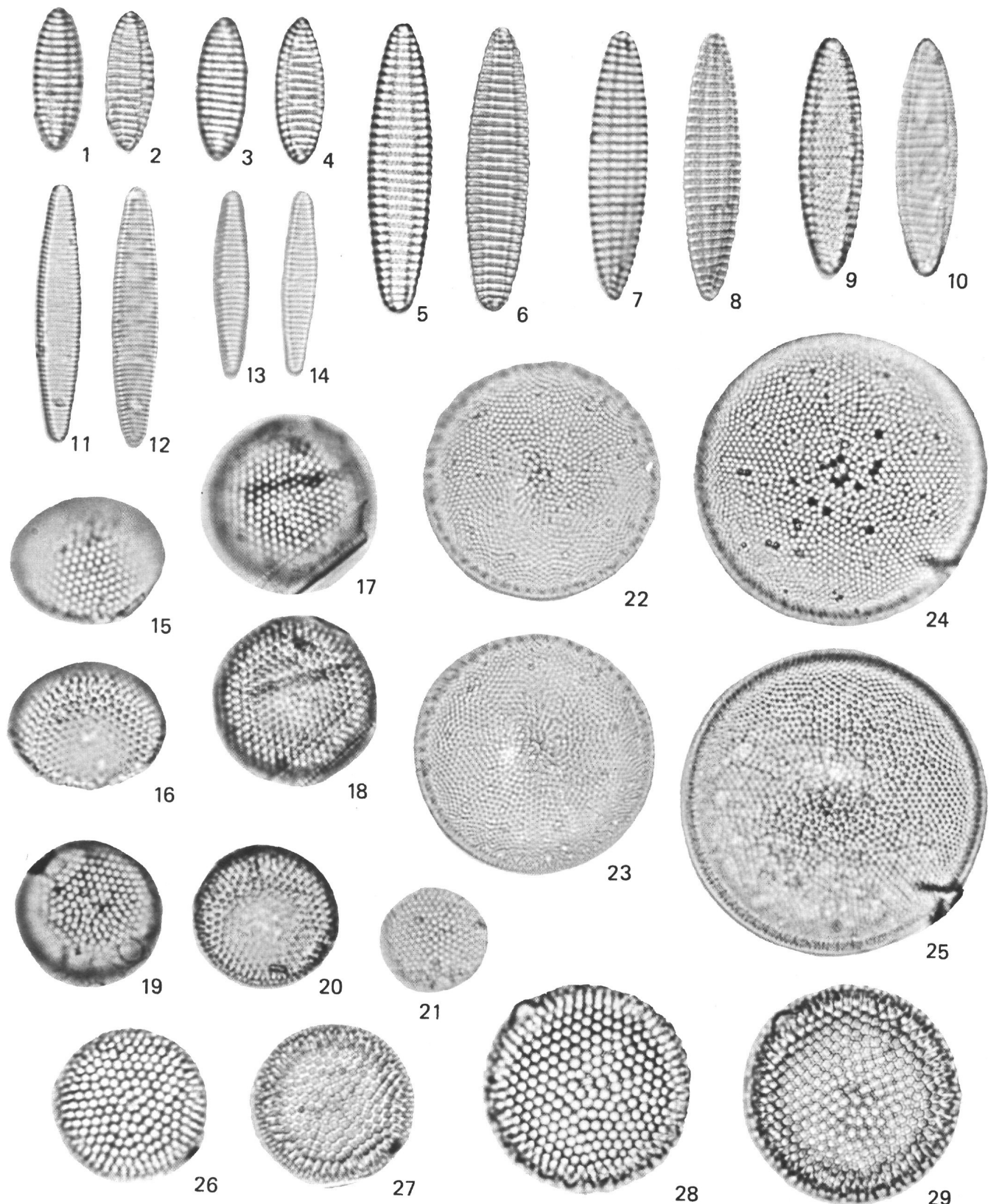

27

28

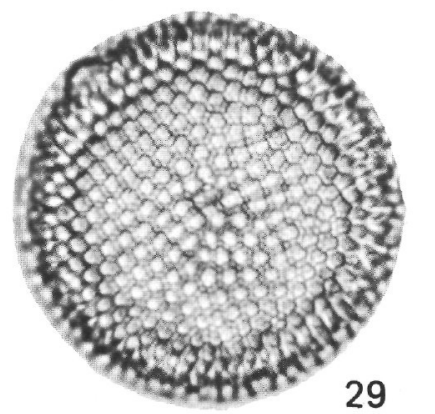

